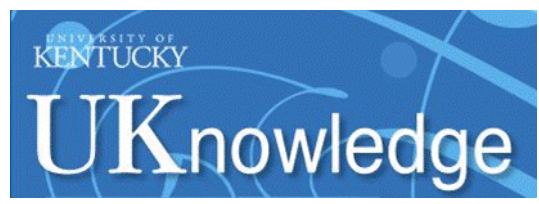

University of Kentucky

UKnowledge

9-20-1999

\title{
Hubble Space Telescope/Faint Object Spectrograph Spectroscopy of Spatially Resolved Narrow-Line Regions in the Seyfert 2 Galaxies NGC 2110 and NGC 5929
}

\author{
Pierre Ferruit \\ University of Maryland \\ Andrew S. Wilson \\ University of Maryland \\ Mark Whittle \\ University of Virginia \\ Chris Simpson \\ National Astronomical Observatory of Japan, Japan \\ John S. Mulchaey \\ Observatories of the Carnegie Institution of Washington \\ Follow this and additional works at: https://uknowledge.uky.edu/physastron_facpub \\ a next page for additional authors \\ Part of the Astrophysics and Astronomy Commons, and the Physics Commons \\ Right click to open a feedback form in a new tab to let us know how this document benefits you. \\ Repository Citation \\ Ferruit, Pierre; Wilson, Andrew S.; Whittle, Mark; Simpson, Chris; Mulchaey, John S.; and Ferland, Gary J., \\ "Hubble Space Telescope/Faint Object Spectrograph Spectroscopy of Spatially Resolved Narrow-Line \\ Regions in the Seyfert 2 Galaxies NGC 2110 and NGC 5929" (1999). Physics and Astronomy Faculty \\ Publications. 112. \\ https://uknowledge.uky.edu/physastron_facpub/112
}

This Article is brought to you for free and open access by the Physics and Astronomy at UKnowledge. It has been accepted for inclusion in Physics and Astronomy Faculty Publications by an authorized administrator of UKnowledge. For more information, please contact UKnowledge@lsv.uky.edu. 
Hubble Space Telescope/Faint Object Spectrograph Spectroscopy of Spatially Resolved Narrow-Line Regions in the Seyfert 2 Galaxies NGC 2110 and NGC 5929

Digital Object Identifier (DOI)

https://doi.org/10.1086/307737

Notes/Citation Information

Published in The Astrophysical Journal, v. 523, no. 1, p. 147-162.

(C) 1999. The American Astronomical Society. All rights reserved. Printed in the U.S.A.

The copyright holder has granted permission for posting the article here.

Authors

Pierre Ferruit, Andrew S. Wilson, Mark Whittle, Chris Simpson, John S. Mulchaey, and Gary J. Ferland 
The Astrophysical JouRnAL, 523:147-162, 1999 September 20

(c) 1999. The American Astronomical Society. All rights reserved. Printed in U.S.A.

\title{
HUBBLE SPACE TELESCOPE/FAINT OBJECT SPECTROGRAPH SPECTROSCOPY OF SPATIALLY RESOLVED NARROW-LINE REGIONS IN THE SEYFERT 2 GALAXIES NGC 2110 AND NGC 5929
}

\author{
PierRe FERruit AND ANDREW S. WILSON ${ }^{2}$ \\ Astronomy Department, University of Maryland, College Park, MD 20742; pierre@astro.umd.edu, wilson@astro.umd.edu \\ MARK WhitTLE \\ Astronomy Department, University of Virginia, Charlottesville, VA 22903; dmwf8@seyfert.astro.virginia.edu \\ CHRIS SIMPSON \\ Subaru Telescope, National Astronomical Observatory of Japan, 650 North A`Ohōkū Place, Hilo, HI 96720; simpson@naoj.org \\ JOHN S. MULChaEY \\ Observatories of the Carnegie Institution of Washington, 813 Santa Barbara Street, Pasadena, CA 91101; mulchaey@pegasus.ociw.edu \\ AND \\ Gary J. FERLAND \\ Department of Physics and Astronomy, University of Kentucky, Lexington, KY 40506; gary@cloud9.pa.uky.edu \\ Received 1999 March 19; accepted 1999 May 6
}

\begin{abstract}
We present the results of UV and optical Hubble Space Telescope/Faint Object Spectrograph spectroscopy of bright, extranuclear regions of line emission in the Seyfert galaxies NGC 2110 and NGC 5929. We have obtained spectra of the brightest region of the "nuclear jet" of NGC 2110 (75 pc from the nucleus) and of the southwest emission-line cloud of NGC 5929 ( $90 \mathrm{pc}$ from the nucleus), in the G130H $(1090-1605 \AA), G 190 \mathrm{H}(1570-2310 \AA), \mathrm{O} 400 \mathrm{H}(3235-4780 \AA)$, and G570H (4570-6820 $)$ configurations. The observed line ratios are compared with the predictions of the two component (matter- and ionization-bounded, MB-IB), central source photoionization models of Binette, Wilson, \& StorchiBergmann and of the fast, photoionizing ("autoionizing”) shock models of Dopita \& Sutherland. In both objects, the significant reddening inferred from the Balmer line ratios and/or its uncertainty limit the utility of the ultraviolet carbon lines C IV $\lambda 1549$ and C III] $\lambda 1909$ for discrimination between the central source and shock-induced photoionization mechanisms. In NGC 2110, shock + precursor models with a shock velocity of $\simeq 400 \mathrm{~km} \mathrm{~s}^{-1}$ provide a better match to the data than the MB-IB models. However, given the simplifying assumptions made in the latter models, photoionization by a central source cannot be ruled out. We investigate whether photoionizing shocks in the emission-line region of NGC 2110 can power the extended, soft X-ray emission north of the nucleus and find that shock velocities higher than $500 \mathrm{~km} \mathrm{~s}^{-1}$ are required. In NGC 5929, the MB-IB models have problems reproducing the strengths of the neon lines, while shock + precursor models with a velocity $\simeq 300 \mathrm{~km} \mathrm{~s}^{-1}$ provide a good match to the data. For both galaxies, the emission-line powers and volumes of the ionized gas inferred from observations imply that both the preshock density $\left(n_{0}\right)$ and magnetic parameter $\left(B_{0} / n_{0}^{1 / 2}\right)$ must be relatively high $\left(n_{0}>10 \mathrm{~cm}^{-3} ; B_{0} / n 1 / 20 \simeq 4 \mu \mathrm{G} \mathrm{cm}^{3 / 2}\right)$ for the photoionizing shock models to be viable.
\end{abstract}

Subject headings: galaxies: individual (NGC 2110, NGC 5929) — galaxies: jets — galaxies: Seyfert — ultraviolet: galaxies $-\mathrm{X}$-rays: galaxies

\section{INTRODUCTION}

Despite extensive studies, the origin of the heating and ionization of the narrow-line region (NLR) and extended narrow-line region (ENLR) of Seyfert galaxies is still controversial. Although photoionization by a compact central source of ionizing radiation undoubtedly occurs in the ENLR of some objects (e.g., in NGC 5252; see Morse et al. 1998), there is a growing line of evidence that other (extended) sources of ionization and heating may be present in the NLR (e.g., in NGC 1068; see Axon et al. 1998) and even in the ENLR (e.g., in Mrk 573; see Ferruit et al. 1999) of Seyfert galaxies.

In the NLR, a close morphological (Haniff, Wilson, \& Ward 1988; Bower et al. 1994, hereafter B94; Capetti et al.

\footnotetext{
${ }^{1}$ Based on observations with the NASA/ESA Hubble Space Telescope obtained at the Space Telescope Science Institute, which is operated by the Association of Universities for Research in Astronomy, Inc., under NASA contract NAS 5-26555.

${ }^{2}$ Adjunct Astronomer, Space Telescope Science Institute.
}

1996; Falcke et al. 1996; Falcke, Wilson, \& Simpson 1998) and kinematical (e.g., Whittle et al. 1986, 1988; Pécontal et al. 1997) association between the ionized gas and the nuclear radio ejecta is observed. This suggests that shocks created by the interaction between the interstellar medium (ISM) of the host galaxy and the radio-emitting material may represent an additional source of ionization and heating (cf. Wilson \& Willis 1980).

Recently, Allen, Dopita, \& Tsvetanov (1998) have compared single-component central source photoionization models, two-component (matter- and ionization-bounded, hereafter MB and IB) central source photoionization models (Binette, Wilson, \& Storchi-Bergmann 1996, hereafter B96; Binette et al. 1997, hereafter B97), and fast photoionizing ("autoionizing") shock models (Dopita \& Sutherland 1995, 1996, hereafter DS95 and DS96) and have shown that they predict very similar optical spectra, but very different ultraviolet (UV) spectra. Allen et al. (1998) and Morse, Raymond, \& Wilson (1996) have outlined the 
importance of the strengths of UV lines such as C III] $\lambda 1909$ and C IV $\lambda 1549$ as shock diagnostics. However, apart from a small number of projects, such as the Hubble Space Telescope (HST) Faint Object Spectrograph (FOS) observations of M87 (Dopita et al. 1997), most of the combined UV and optical spectroscopic studies of Seyfert galaxies consist of observations through apertures centered on the nucleus. Because nuclear spectra represent a superposition along the line of sight of many gaseous components with very different physical conditions (in particular, a wide range of density), their interpretation is usually ambiguous.

For these reasons, we have performed off-nuclear UV and optical spectroscopy of the two Seyfert galaxies NGC 2110 and NGC 5929 with the FOS on board HST. For each galaxy, spectra have been obtained of a bright extranuclear emission-line region that is close to a radio jet or lobe. These locations were chosen to maximize the chance that the off-nuclear gas is heated and/or ionized by the radio ejecta. In this paper, we report the results of these observations and compare the observed UV and optical line ratios with the predictions of the MB-IB central source photoionization models (B96, B97) and of the photoionizing shock models (DS95, DS96). In NGC 2110, we also discuss whether fast shocks can power the extended soft X-ray emission detected in this galaxy (Weaver et al. 1995).

NGC 2110 is an elliptical or S0 galaxy first studied as an $\mathrm{X}$-ray source and identified as a Seyfert 2 galaxy in the optical spectroscopic follow-up (Bradt et al. 1978; see also McClintock et al. 1979 and Shuder 1980). Since then, ground-based optical spectroscopic and narrowband imaging studies have revealed an asymmetric emission-line region extending over $\simeq 10^{\prime \prime}$ (Wilson, Baldwin, \& Ulvestad 1985; Pogge 1989; Haniff, Ward, \& Wilson 1991), and VLA observations have found an S-shaped jetlike radio source extending over $\simeq 5^{\prime \prime}$ (Ulvestad \& Wilson 1984; Nagar et al. 1999). High spatial resolution [O III] and $\mathrm{H} \alpha+[\mathrm{N} \mathrm{II}] H S T$ images obtained by Mulchaey et al. (1994, hereafter M94)

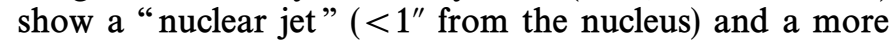
extended $(\simeq 4$ ") "S-shaped" structure to the north of the nucleus. Extended soft X-ray emission is also detected 4" north of the nucleus, in the vicinity of the "S-shaped" emission-line feature (Weaver et al. 1995).
NGC 5929 is a member of an interacting pair with NGC 5930 (Arp 90; see Arp 1966) and hosts a Seyfert 2 nucleus (e.g., Wilson \& Keel 1989 and references therein). It displays an extended emission-line region with two main components, one blueshifted and one redshifted, located northeast and southwest of the nucleus, respectively (Whittle et al. 1986; Wilson \& Keel 1989; B94; Ferruit et al. 1997b). Radio observations show a triple radio source with two lobes straddling an unresolved nuclear component (Ulvestad \& Wilson 1984; Wilson \& Keel 1989; Su et al. 1996). Comparison between the radio maps and the emission-line observations reveals a close morphological and kinematical association between the ionized gas and the radio-emitting material (Whittle et al. 1986; B94; Su et al. 1996; Lattanzi, Capetti, \& Macchetto 1997; Ferruit et al. 1997b).

Throughout this paper, we will use systemic heliocentric velocities of $2335 \pm 20 \mathrm{~km} \mathrm{~s}^{-1}$ (stellar Mg I $b$ absorption lines; Nelson \& Whittle 1995) and $2492 \pm 8 \mathrm{~km} \mathrm{~s}^{-1}$ (stellar Mg I $b$ and Ca II absorption lines; Nelson \& Whittle 1995) for NGC 2110 and NGC 5929, respectively. This gives velocities relative to the cosmic microwave background of $2404 \mathrm{~km} \mathrm{~s}^{-1}$ (NGC 2110, +69.5 $\mathrm{km} \mathrm{s}^{-1}$ correction) and $2585 \mathrm{~km} \mathrm{~s}^{-1}$ (NGC 5929, $+92.7 \mathrm{~km} \mathrm{~s}^{-1}$ correction), as derived using the $\mathrm{NED}^{3}$ on-line velocity calculator. For NGC 2110 [NGC 5929], this corresponds to a distance and a linear scale of $32 \mathrm{Mpc}[34 \mathrm{Mpc}]$ and $150 \mathrm{pc} \operatorname{arcsec}^{-1}[160$ $\left.\mathrm{pc}^{-1}\right]$, respectively $\left(H_{0}=75 \mathrm{~km} \mathrm{~s}^{-1} \mathrm{Mpc}^{-1}, q_{0}=0\right)$.

\section{OBSERVATIONS}

These off-nuclear spectra of NGC 2110 and NGC 5929 were obtained with the FOS aboard HST on 1996 March 23 (NGC 2110) and 1996 October 12 (NGC 5929). The journal of observations is given in Table 1 . Successive peakups in progressively smaller apertures $(0,86,0$, 43 , and 0.26 diameter), followed by an acquisition image, were used to

\footnotetext{
${ }^{3}$ The NASA/IPAC Extragalactic Database (NED) is operated by the Jet Propulsion Laboratory, California Institute of Technology, under contract with the National Aeronautics and Space Administration.
}

TABLE 1

FOS SPECTRA Discussed IN This PAPER

\begin{tabular}{|c|c|c|c|c|c|c|}
\hline Object & Exposure & $t_{i}^{\mathrm{a}}$ & Grating & $\begin{array}{c}\text { Range } \\
(\AA)\end{array}$ & $\begin{array}{c}\text { Sampling } \\
(\AA)\end{array}$ & $\begin{array}{l}\text { Resolution } \\
\text { (§) }\end{array}$ \\
\hline NGC $2110 \ldots . .$. & Y3470106T & 300 & G570H & $4570-6820$ & 1.1 & $5.24^{\mathrm{b}}$ \\
\hline NGC $2110 \ldots . .$. & Y3470107T & 600 & $\mathrm{G} 400 \mathrm{H}$ & $3235-4780$ & 0.75 & $3.6^{\mathrm{b}}$ \\
\hline NGC $2110 \ldots . .$. & Y3470108T & 300 & G190H & $1570-2310$ & 0.36 & $1.7^{\mathrm{b}}$ \\
\hline NGC $2110 \ldots . .$. & Y347010BT & 820 & $\mathrm{G} 130 \mathrm{H}$ & $1090-1605$ & 0.25 & $1.1^{\mathrm{c}}$ \\
\hline NGC $2110 \ldots . .$. & Y347010AT & 1280 & $\mathrm{G} 130 \mathrm{H}$ & $1090-1605$ & 0.25 & $1.1^{\mathrm{c}}$ \\
\hline NGC $2110 \ldots \ldots$ & Y347010CT & 1400 & $\mathrm{G} 130 \mathrm{H}$ & $1090-1605$ & 0.25 & $1.1^{\mathrm{c}}$ \\
\hline NGC $5929 . . . .$. & Y3470301T & 300 & G570H & $4570-6820$ & 1.1 & $5.24^{\mathrm{b}}$ \\
\hline NGC $5929 . . . .$. & Y3470302T & 600 & $\mathrm{G} 400 \mathrm{H}$ & $3235-4780$ & 0.75 & $3.6^{\mathrm{b}}$ \\
\hline NGC $5929 . . . .$. & Y3470303T & 330 & G190H & $1570-2310$ & 0.36 & $1.7^{\mathrm{b}}$ \\
\hline NGC $5929 . . . .$. & Y3470305T & 1500 & $\mathrm{G} 130 \mathrm{H}$ & $1090-1605$ & 0.25 & $1.3^{\mathrm{c}}$ \\
\hline
\end{tabular}

NoTE.-All the exposures were taken through the (0"43 post-COSTAR diameter). All the grating configurations except G130H used the AMBER (FOS/RD) detector.

${ }^{a}$ Exposure time in seconds.

${ }^{\mathrm{b}}$ Upper limit derived assuming a 1.20 diode FWHM (aperture filled with a uniform source; Keyes et al. 1995).

${ }^{\mathrm{c}}$ Derived from single-Gaussian fitting of the geocoronal Ly $\alpha$. 
find the location of the continuum ${ }^{4}$ peak of each galaxy. Special care was given to the acquisition procedure for NGC 5929, the central regions of which are divided by a dust lane running northeast-southwest (B94). For this galaxy, the acquisition image obtained in the first visit to the galaxy was carefully examined to confirm that the successive peak-up procedure had correctly located the continuum peak. The actual spectroscopic observations were performed in the second visit several weeks later, when the telescope pointing was first returned to the continuum peak as obtained by the acquisition in the first visit. For both galaxies, once the continuum peak had been located, the aperture was then shifted to the bright off-nuclear emissionline features seen in HST wide field planetary camera (WF/PC) pre-COSTAR images (NGC 2110: M94; NGC 5929: B94). The following offsets from the continuum peak were applied: $\Delta \alpha=-0$ "'.19 and $\Delta \delta=+0$ ".46 (NGC 2110); $\Delta \alpha=-0.38$, and $\Delta \delta=-0$ ".41 (NGC 5929). The galaxy spectra were then taken through the 0 ". 43 diameter circular aperture, using the G130H (1090-1605 $),$ G190H (1570$2310 \AA), \mathrm{G} 400 \mathrm{H}(3235-4780 \AA)$, and G570H (4570-6820 ̊) configurations. The location and size of the aperture for each object are shown in Figure 1, superimposed on the $H S T$ [O III $]+\mathrm{H} \beta$ images, while the accuracy in their positioning is discussed in $\S 3.2$.

\section{DATA REDUCTION}

\subsection{Basic Data Reduction}

The data reduction has been performed in a standard way using the IRAF CALFOS task and the best available reference files associated with the observation dates. For the G130H configuration, we have corrected the spectra for the reported change of sensitivity in the $1250-1600 \AA$ range (which is not accounted for by the CALFOS task). The correction factors longward of $1250 \AA$ were 0.9775 (NGC 2110) and 0.9625 (NGC 5929), corresponding to a sensi-

\footnotetext{
${ }^{4}$ The acquisition procedure has been performed with the FOS/RD detector without filter. The corresponding passband was $1700-8500 \AA$, with a rapidly decreasing sensitivity beyond $4000 \AA$ (Keyes et al. 1995).
}

TABLE 2

\begin{tabular}{|c|c|c|c|c|}
\hline \multirow[b]{2}{*}{ LINES } & \multicolumn{2}{|c|}{ NGC 2110} & \multicolumn{2}{|c|}{ NGC 5929} \\
\hline & FOS & $\mathrm{WF} / \mathrm{PC}^{\mathrm{a}}$ & FOS & $\mathrm{WF} / \mathrm{PC}^{\mathrm{b}}$ \\
\hline $\mathrm{O}$ III] $+\mathrm{H} \beta \ldots \ldots$ & 49 & 20 & 22 & 15 \\
\hline $\mathrm{N}$ II $]+\mathrm{H} \alpha \ldots \ldots$ & 62 & 49 & 38 & 30 \\
\hline
\end{tabular}

Note.-Units: $10^{-15} \mathrm{ergs} \mathrm{s}^{-1} \mathrm{~cm}^{-2}$. Derived from our FOS spectra and from the WF/PC deconvolved emission-line images after integration over a 0 ".43 diameter aperture centered at the expected FOS aperture locations.

a From Mulchaey et al. 1994.

${ }^{b}$ From Bower et al. 1994.

tivity increase of $0.25 \%$ per month between 1995 July 1 and the date of observation. In addition, a linear ramp has been applied to the sensitivities between 1200 and $1250 \AA$, providing a smoother transition between the corrected and uncorrected wavelength domains. The three $\mathrm{G} 130 \mathrm{H}$ spectra obtained for NGC 2110 (see Table 1) have been averaged to increase the signal-to-noise ratio. Further processing (see below) has been performed under the ESO/MIDAS software environment.

\subsection{Aperture Location}

The locations of the apertures have been checked by comparing the $[\mathrm{O} \mathrm{III}]+\mathrm{H} \beta$ and $[\mathrm{N} \mathrm{II}]+\mathrm{H} \alpha$ fluxes derived from our spectra with those measured from the deconvolved $H S T / \mathrm{WF} / \mathrm{PC}$ emission-line images (NGC 5929: B94; NGC 2110: M94) after integration over a 0"43 diameter circular aperture at the intended aperture position. As shown in Table 2, the fluxes derived from the WF/PC images of NGC 2110 are systematically lower than our FOS fluxes by $60 \%(20 \%)$ for $[\mathrm{O}$ III $]+\mathrm{H} \beta([\mathrm{N} \mathrm{II}]+\mathrm{H} \alpha)$. If the $\mathrm{WF} / \mathrm{PC}$ fluxes are correct, this discrepancy would imply that the FOS aperture was only 0 ."2-0.3 from the nucleus, closer than intended. M94 report that the fluxes from their deconvolved images are systematically lower than groundbased measurements by up to $30 \%$ for $[\mathrm{O}$ III] $+\mathrm{H} \beta$.
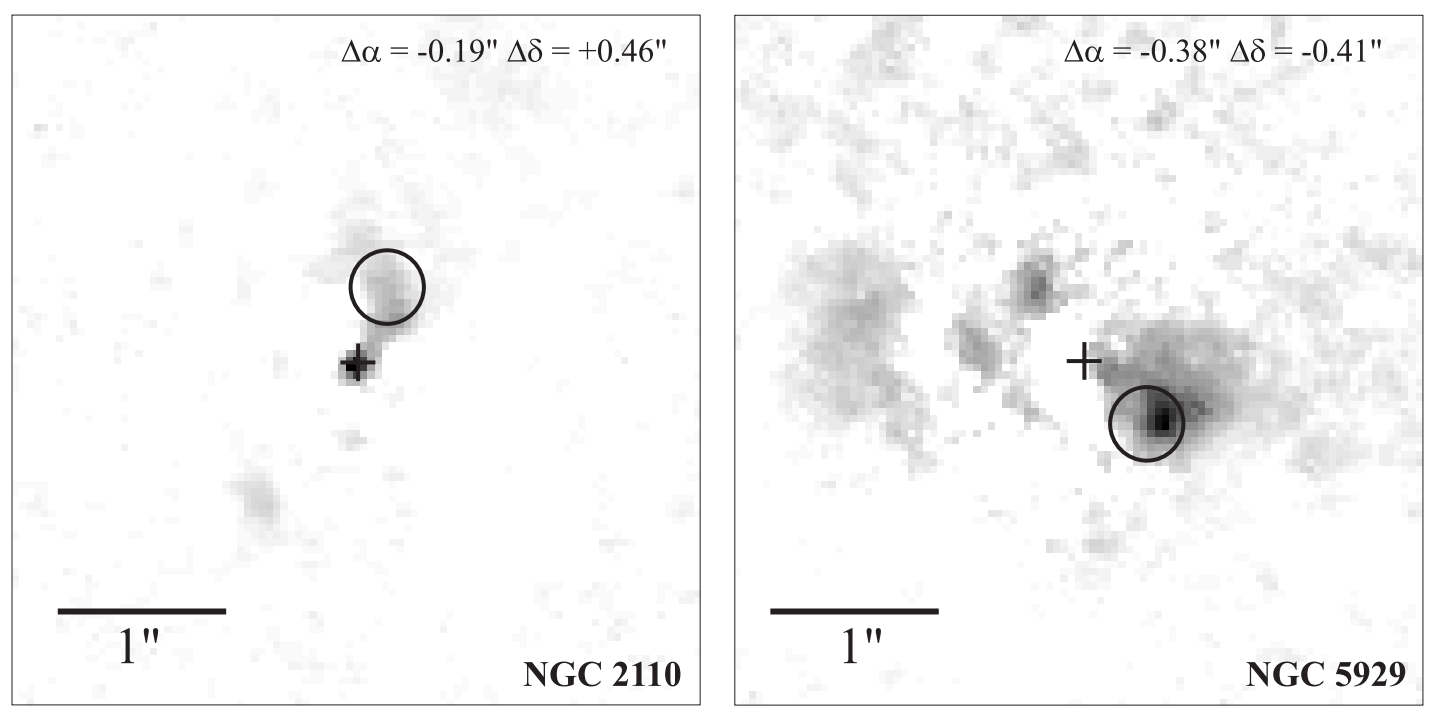

FIG. 1.-Locations and size of the FOS 0"43 diameter aperture (circle) superimposed on the $H S T / \mathrm{WF} / \mathrm{PC} \mathrm{H} \beta+[\mathrm{O}$ III] deconvolved images of NGC 2110 (left, from M94) and NGC 5929 (right, from B94). The cross marks the position of the continuum peak, which in the case of NGC 5929 is not the position of the true nucleus (Lattanzi et al. 1997). 
However, increasing the WF/PC image fluxes by $30 \%$ and comparing again with our FOS measurements does not imply an aperture location significantly closer to its intended position, as the emission-line nucleus is strongly peaked in NGC 2110 (off-nuclear extensions in the WF/PC deconvolved $[\mathrm{O} \mathrm{III}]+\mathrm{H} \beta$ image have fluxes integrated over a 0.43 aperture lower than $2.6 \times 10^{-14} \mathrm{ergs} \mathrm{s}^{-1} \mathrm{~cm}^{-2}$, i.e., $50 \%$ lower than the observed FOS one, no matter where the aperture is located). We believe that the most likely cause of this discrepancy is that the fluxes derived from the pre-COSTAR, deconvolved WF/PC images are unreliable, but we cannot exclude the possibility that the FOS aperture was closer to the nucleus of NGC 2110 than intended.

For NGC 5929, both [O III $]+\mathrm{H} \beta$ and $[\mathrm{N} \mathrm{II}]+\mathrm{H} \alpha$ FOS fluxes are higher than those derived from the deconvolved HST/WF/PC images (B94; see Table 2) but are consistent with the aperture being within 0 ".2 of its desired location. This location is the southwest off-nuclear line-emitting cloud (cloud A; B94).

\subsection{Continuum Subtraction}

Comparison of a shifted and reddened template E2-E5 galaxy spectrum from Bica et al. (in Leitherer et al. 1996) with the spectrum of NGC 2110 shows that all the expected absorption features are well below the detection level in the FOS spectra and should not be significant even in our higher spectral resolution optical spectra. This is confirmed by visual inspection of wavelength areas in our spectra where strong absorption lines are expected (see Table 2 in Shuder 1980); the late-type stellar population of this galaxy (Shuder 1980) suggests that dilution of the Balmer emission by Balmer absorption features is negligible. This comparison indicates that no correction for stellar absorption lines is necessary. A similar inspection of the spectra of NGC 5929 leads to the same conclusion although stellar features are weakly seen in this object. Subtraction of the underlying continuum in both objects has, therefore, been performed locally using a straight-line fit to the continuum on each side of the emission line.

\subsection{Measurements of the Line Fluxes and Velocity Centroids}

The integrated fluxes of isolated lines have been measured using the INTEGRATE/LINE task of the ESO/ MIDAS software, the continuum being estimated using line-free regions on each side of the line. This method does not require any assumption about the shape of the line profile, but cannot be used for either the [S II] $\lambda \lambda 6717,6731$ doublet, or the $[\mathrm{N}$ II $] \lambda \lambda 6548,6583$ and $\mathrm{H} \alpha$ lines, which are blended. The procedure used to disentangle the $[\mathrm{N}$ II] and $\mathrm{H} \alpha$ lines is described in $\S 3.6$. For the [S II] doublet, the fluxes have been measured with the FIT/SPEC task (Rousset 1992) using a single-Gaussian fit to the lines. No multiple Gaussian fit was necessary since, given our error bars, the total [S II] $\lambda \lambda 6717,6731$ flux derived from singleGaussian fitting agrees with that derived by integrating over the doublet.

Deblending of the $\left[\mathrm{O}_{\mathrm{I}}\right] \lambda 6300$ and $[\mathrm{S}$ III] $\lambda 6310$ lines in NGC 2110 has been performed using single-Gaussian fitting and assuming that the weaker [S III] line has the same velocity and width as the $[\mathrm{O} \mathrm{I}]$ line. These assumptions are questionable but were necessary to stabilize the fit. The wavelengths of unblended or deblended lines have been obtained either by fitting a single Gaussian (for lines without significant departure from a Gaussian profile) or by taking the centroid of the line $(\mathrm{H} \beta,[\mathrm{O} \mathrm{III}],[\mathrm{N} \mathrm{II}]$, and $\mathrm{H} \alpha$ lines).

\subsection{Reliability of the Measurements}

To estimate the accuracy of the line flux and velocity measurements, all the measurements (for each line or set of blended lines) have been repeated 500 times using artificial spectra consisting of the sum of a synthetic, noise-free spectrum (from the result of a multiple Gaussian fitting of the lines) and noise, the magnitude of which was derived from the residuals to the multiple Gaussian fitting (or to the single-Gaussian fitting for the [S II] doublet). The error bar was conservatively set as to include $90 \%$ of these 500 measurements. Upper limits on the integrated fluxes of undetected lines have been set by using 3 times the noise rms as an upper limit on the line peak flux and typical line widths determined on other (detected) emission lines.

The total error of the velocity of a line was taken as the quadratic sum of the error from the velocity measurement and the intrinsic error from systematic instrumental effects in the FOS spectrograph ( $20 \mathrm{~km} \mathrm{~s}^{-1}$; Keyes et al. 1995). As no wavelength calibration exposures were taken at the same time as the science exposures, differences of up to $\simeq 0.35$ diodes (Keyes et al. 1995) can be found between different grating configurations owing to nonrepeatability of the filter-grating wheel position. This prevents us from obtaining any accurate absolute velocity measurements. Given these uncertainties, no correction for the motion of $H S T$ has been attempted (i.e., the velocities are not heliocentric).

For the line profile comparisons, no correction for instrumental broadening has been applied since this correction depends strongly on the grating configuration and on the way the source fills the aperture (Keyes et al. 1995), which itself can vary systematically from the UV to the optical owing to reddening effects. Therefore, a quantitative comparison of line profiles has been possible only within spectra obtained with a given grating.

\subsection{Deblending of the $[\mathrm{N} \mathrm{II}]$ and $\mathrm{H} \alpha$ Lines}

The $[\mathrm{N}$ II] $\lambda \lambda 6548,6583$ and $\mathrm{H} \alpha$ lines have been deblended to recover their profiles. A first try, fitting each line with a single-Gaussian profile, failed to recover either the total flux (the sum of all three lines) or the theoretical ratio of 2.94 between the two nitrogen lines. We therefore used two Gaussians to model each line, checking afterward that the final fluxes were consistent with the total flux derived from integration over the whole blend. The profile of $[\mathrm{N} \mathrm{II}] \lambda 6583$ was first fitted with two Gaussians of different widths and velocities using only the red side and the core of the line, both of which are essentially free of contamination by $\mathrm{H} \alpha$. These Gaussian fits were then used to model the [N II] $\lambda \lambda 6548,6583$ doublet, assuming the theoretically expected flux ratio of 2.94 between the two lines, and this model was subtracted from the continuum-subtracted spectrum, yielding the $\mathrm{H} \alpha$ line profile. It should be noted that this method provides only loose constraints on the blue side of [N II] $\lambda 6583$, so our estimation of the red side of the $\mathrm{H} \alpha$ line profile is not very reliable.

The results of this procedure are shown in Figure 2. As for the other line flux measurements (see $\S 3.5$ ), an estimate of the errors on the profile has been computed by repeating this procedure 500 times using artificial $[\mathrm{N} \mathrm{II}]+\mathrm{H} \alpha$ spectra consisting of the sum of a synthetic noise-free spectrum 

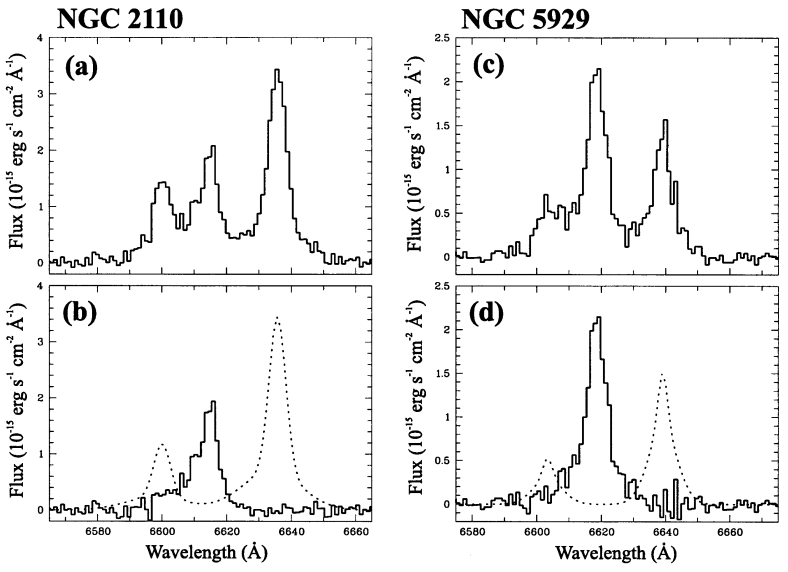

FIG. 2.-Left: NGC 2110. (a) Plot of the continuum-subtracted G570H configuration spectrum in the $[\mathrm{N} \mathrm{II}]+\mathrm{H} \alpha$ region. (b) Plot of the $\mathrm{H} \alpha$ line profile (solid line) obtained by subtracting a synthetic [N II] doublet (dotted line; see $\S 3.6$ ) from the continuum-subtracted G570H spectrum. Right: NGC 5929. (c) Plot of the continuum-subtracted G570H configuration spectrum in the $[\mathrm{N}$ II $]+\mathrm{H} \alpha$ region. (d) Plot of the $\mathrm{H} \alpha$ line profile (solid line) obtained by subtracting a synthetic $[\mathrm{N} \mathrm{II}]$ doublet (dotted line; see $\S 3.6)$ from the continuum-subtracted $\mathrm{G} 570 \mathrm{H}$ spectrum.

(derived from a multiple Gaussian fitting of the lines) and noise (derived as described in $\S 3.5$ ). The error bar at each spectral pixel on the profile was then set to the $90 \%$ confidence level. Systematic errors in the line profiles, due to uncertainty in the deblending described above, are not included in these errors. The error bars will be used later (§ 4.3) when evaluating the differences between the profiles of different lines.

\subsection{Reddening}

The total (internal plus Galactic) reddenings toward the ionized gas in NGC 5929 and NGC 2110 have been estimated by comparing the observed Balmer decrements with the expected Case B values. We have used an analytical fit to the extinction law from Cardelli, Clayton, \& Mathis (1989) with $R_{V}=A_{V} / E_{B-V}=3.1$ (standard value for the diffuse interstellar medium; Cardelli et al. 1989). Note that this curve includes the $2175 \AA$ extinction bump, the additional reddening of which affects the $\mathrm{C}$ III] $\lambda 1909$ lines.

For NGC 2110, all the nominal reddening values derived from the Balmer line ratios indicate a total obscuration of $A_{V} \simeq 1.2-1.5 \mathrm{mag}$ (see Table 3) consistent with the $F(5461$ $\AA$ ) $/ F(7164 \AA$ A) continuum flux ratio of $\simeq 0.7$ (which corresponds to $A_{V} \simeq 1.4 \mathrm{mag}$ ) measured by M94 in this galaxy (excluding the highly reddened nucleus and dust lane, which are not included in our aperture). The contribution of Galactic obscuration to this total is estimated to be $A_{V}=$ 1.05 mag (M94, from the $\mathrm{H}$ I measurements by Burstein \& Heiles 1982). These values are much smaller than the estimate of the total obscuration of $A_{V}=4.7 \pm 2.2 \mathrm{mag}$ $\left(E_{B-V}=1.5 \pm 0.7 \mathrm{mag}\right)$, obtained recently by StorchiBergmann et al. (1999) from the $\mathrm{Br} \gamma / \mathrm{Pa} \beta$ ratio, but their aperture included the reddened nucleus (M94). In the following, we will provide reddening-corrected line fluxes for three values of $A_{V}: 1.05$ (Galactic extinction only), 1.2, and $1.4 \mathrm{mag}$.

Despite the low level of Galactic obscuration toward NGC $5929\left(A_{V}=0.0465 \mathrm{mag}\right.$; Burstein \& Heiles 1984), significant obscuration $\left(A_{V}=1.5_{-0.9}^{+0.7} \mathrm{mag}\right)$ is found from the $\mathrm{H} \alpha / \mathrm{H} \beta$ ratio, which suggests the presence of internal extinc-
TABLE 3

Estimates OF the Total (Galactic plus Internal) REDDENING TOWARD THE IONIZED GAS IN NGC 2110 AND NGC 5929

\begin{tabular}{cccc}
\hline \hline \multicolumn{4}{c}{ NGC 2110} \\
\hline Ratio & Observed & Expected $^{\mathrm{a}}$ & $A_{V}{ }^{\mathrm{b}}(\mathrm{mag})$ \\
\hline $\mathrm{H} \delta / \mathrm{H} \beta \ldots \ldots$ & $0.19 \pm 0.08$ & 0.259 & $1.5_{-1.5}^{+2.2}$ \\
$\mathrm{H} \gamma / \mathrm{H} \beta \ldots \ldots$ & $0.37 \pm 0.13$ & 0.468 & $1.3_{-1.3}^{+2.8}$ \\
$\mathrm{H} \alpha / \mathrm{H} \beta \ldots \ldots$ & $4.2 \pm 1.6$ & 2.86 & $1.3_{-1.3}^{+1.0}$ \\
\hline \hline \multicolumn{4}{c}{ NGC 5929} \\
\hline Ratio & Observed & Expected $^{\mathrm{a}}$ & $A_{V}^{\mathrm{b}}(\mathrm{mag})$ \\
\hline $\mathrm{H} \delta / \mathrm{H} \beta \ldots \ldots$ & $0.25 \pm 0.08$ & 0.259 & $<1.5$ \\
$\mathrm{H} \gamma / \mathrm{H} \beta \ldots \ldots$ & $0.39 \pm 0.11$ & 0.468 & $1.1_{-1.1}^{+2.0}$ \\
$\mathrm{H} \alpha / \mathrm{H} \beta \ldots \ldots$ & $4.7 \pm 1.2$ & 2.86 & $1.5_{-0.9}^{+0.7}$
\end{tabular}

NotE.-Derived using the Balmer line ratios $\mathrm{H} \delta / \mathrm{H} \beta$, $\mathrm{H} \gamma / \mathrm{H} \beta$ and $\mathrm{H} \alpha / \mathrm{H} \beta$.

${ }^{a}$ Case B, $T_{e}=10^{4} \mathrm{~K}, n_{e}=10^{2} \mathrm{~cm}^{-3}$ (Osterbrock 1989).

b The error bars correspond to the $90 \%$ confidence level.

tion. The other Balmer lines (see Table 3) provide us with only upper limits. No additional constraints on $A_{V}$ can be set using previously published Balmer decrement measurements from ground-based observations (e.g., $\mathrm{H} \alpha / \mathrm{H} \beta=5.93$, yielding $A_{V} \simeq 2.3 \mathrm{mag}$; Keel et al. 1985) as they all include the dust lane $\left(A_{V} \simeq 2.5 \mathrm{mag}\right.$; B94) in their aperture. Recently, Cole et al. (1998) have determined an upper limit to the hydrogen column density to the southwest radio lobe of NGC 5929 (which coincides with the cloud A we are studying) using $21 \mathrm{~cm}$ observations. They find $N_{\mathrm{H}}<2$ $\times 10^{20}\left(T_{S} / 100 \mathrm{~K}\right) \mathrm{cm}^{-2}$, where $T_{S}$ is the spin temperature. For a spin temperature of $100 \mathrm{~K}$ and the normal relationship between obscuration and hydrogen column- $A_{V}$ (mag) $=5 \times 10^{22} N_{\mathrm{H}}\left(\mathrm{cm}^{-2}\right)$-we find $A_{V}<0.1 \mathrm{mag}$. Such a low obscuration is also suggested by the detection in our G400H spectrum of NGC 5929 of the H9 to H11 Balmer lines, the nominal, measured flux ratios to $\mathrm{H} \beta$ of which are consistent with the Case B values. Thus, these different estimates of $A_{V}$ are inconsistent, and we have, therefore, decided to compute the line fluxes for four different values of $A_{V}: \simeq 0 \mathrm{mag}$ (suggested by the $21 \mathrm{~cm}$ observations and the higher order Balmer lines), $0.6 \mathrm{mag}$ (lower limit from the $\mathrm{H} \alpha / \mathrm{H} \beta$ ratio), $0.9 \mathrm{mag}$ (intermediate value), and $1.2 \mathrm{mag}$ (upper limit on the stellar continuum reddening away from the dust lane from B94).

The use of Case $B$ values for the intrinsic $\mathrm{H} \alpha / \mathrm{H} \beta$ ratio is debatable. Indeed, this ratio is believed to be higher in the NLR of Seyfert galaxies (3.0-3.1; Halpern \& Steiner 1983; Gaskell \& Ferland 1984) than the values computed for Case B (2.7-3.0). However, using a value of 3.0 instead of 2.86 yields a decrease of only $0.1-0.2 \mathrm{mag}$ for the $A_{V}$ derived from the $\mathrm{H} \alpha / \mathrm{H} \beta$ ratio. Only if $\mathrm{H} \alpha / \mathrm{H} \beta$ is as high as 3.4 , as suggested by Binette et al. (1990), will the nominal reddening values derived from the observed ratios be significantly lowered. However, given our error bars, this would not alter the conclusions of the previous paragraphs.

\section{RESULTS}

\subsection{NGC 2110: Line Ratios}

The FOS spectra of NGC 2110 are shown in Figure 3 and the measured fluxes listed in Table 4. High-excitation 

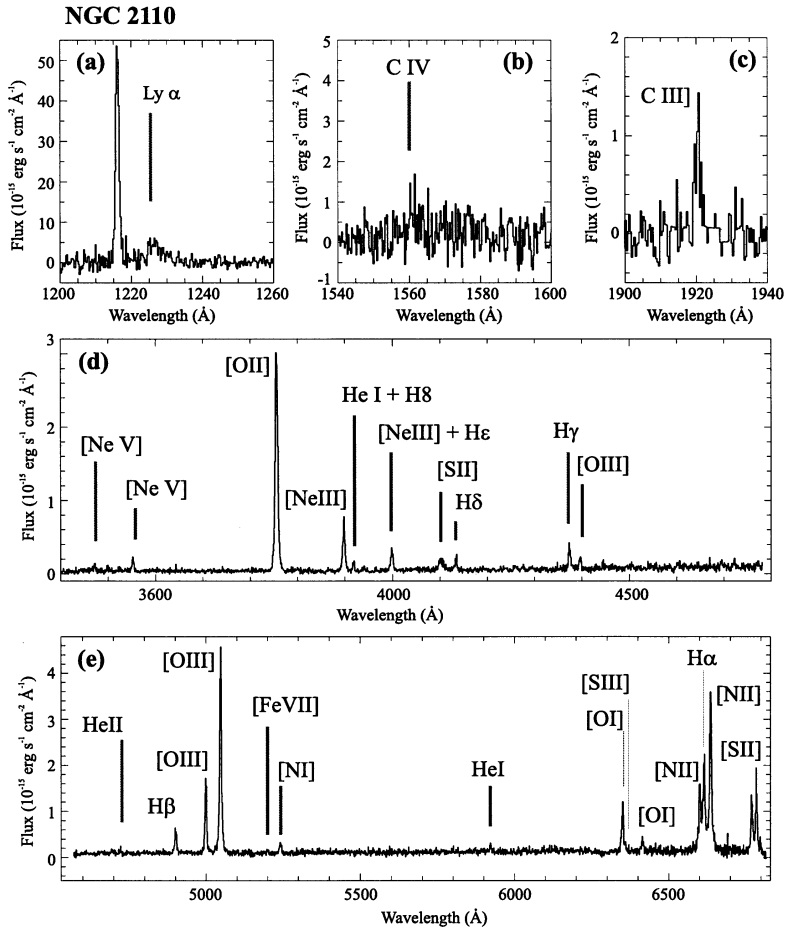

FIG. 3.- HST FOS spectra of NGC 2110. (a) Part of the G130H configuration spectrum displaying strong geocoronal Ly $\alpha \lambda 1215.67$ and faint Ly $\alpha$ from the galaxy. (b) Part of the G130H configuration spectrum with the expected location of the C IV $\lambda 1549$ lines marked with a vertical line. A weak feature is present at this wavelength, but the signal-to-noise ratio is too low to discriminate it from noise. (c) Part of the G190H configuration spectrum displaying the $\mathrm{C} \mathrm{III]} \mathrm{lines.} \mathrm{(d)} \mathrm{G} 400 \mathrm{H}$ configuration spectrum. (e) $\mathrm{G} 570 \mathrm{H}$ configuration spectrum.

lines such as $[\mathrm{Ne} \mathrm{v}] \lambda \lambda 3346,3426$ are clearly detected as well as the temperature diagnostic line $[\mathrm{O}$ III] $\lambda 4363$. Assuming a reddening of $A_{V}=1.05$ mag (Galactic reddening only) and the low-density regime (no collisional deexcitation of $[\mathrm{O} \mathrm{III}] \lambda 5007)$, we derive an $[\mathrm{O} \mathrm{III}]$ temperature of $17,400_{-1,600}^{+2,600} \mathrm{~K}$. The $[\mathrm{S} \mathrm{II}]$ line ratios indicates a density of $1200_{-300}^{+500} \mathrm{~cm}^{-3}$ and a temperature of $13,900_{-2,000}^{+3,000} \mathrm{~K}\left(A_{V}=1.05 \mathrm{mag}\right.$; any increase in reddening will yield an increase in temperature). Also for $A_{V}=1.05$ mag and a density of $1200 \mathrm{~cm}^{-3}$, the derived upper limit on the $[\mathrm{N} \mathrm{II}] \lambda 5755 /[\mathrm{N}$ II $] \lambda \lambda 6548,6583$ ratio $(<0.029)$ indicates a temperature less than $15,500 \mathrm{~K}$, lower than the nominal temperature obtained from the $[\mathrm{O}$ III] lines. Similar differences between the $[\mathrm{N}$ II] and $[\mathrm{O}$ III] temperatures are found in the extended gas of several active galaxies (Wilson, Binette, \& Storchi-Bergmann 1997). Last, the detection of the [S III] $\lambda 6310$ line (equivalent to the [O III] $\lambda 4363$ line for the [S III] near-infrared doublet) is suggestive of a relatively high temperature in the [S III]emitting zone.

\subsection{NGC 5929: Line Ratios}

The FOS spectra of NGC 5929 are shown in Figure 4, and the measured fluxes are listed in Table 5. The observed spectrum is of low excitation with strong Balmer lines, a low [O III] $\lambda 5007 / \mathrm{H} \beta$ ratio and strong [O II] $\lambda 3727$ lines. The density obtained from the [S II] $\lambda \lambda 6717,6731$ lines is $320_{-150}^{+220} \mathrm{~cm}^{-3}$. Our G400H spectrum of NGC 5929 also displays unusually strong $[\mathrm{S}$ II] $\lambda \lambda 4069,4077$ lines. Without any reddening correction and for a density of $320 \mathrm{~cm}^{-3}$, the [S II] $\lambda \lambda 4069,4077 /[\mathrm{S} \mathrm{II}] \lambda \lambda 6717,6731$ ratio $(0.14 \pm 0.04)$
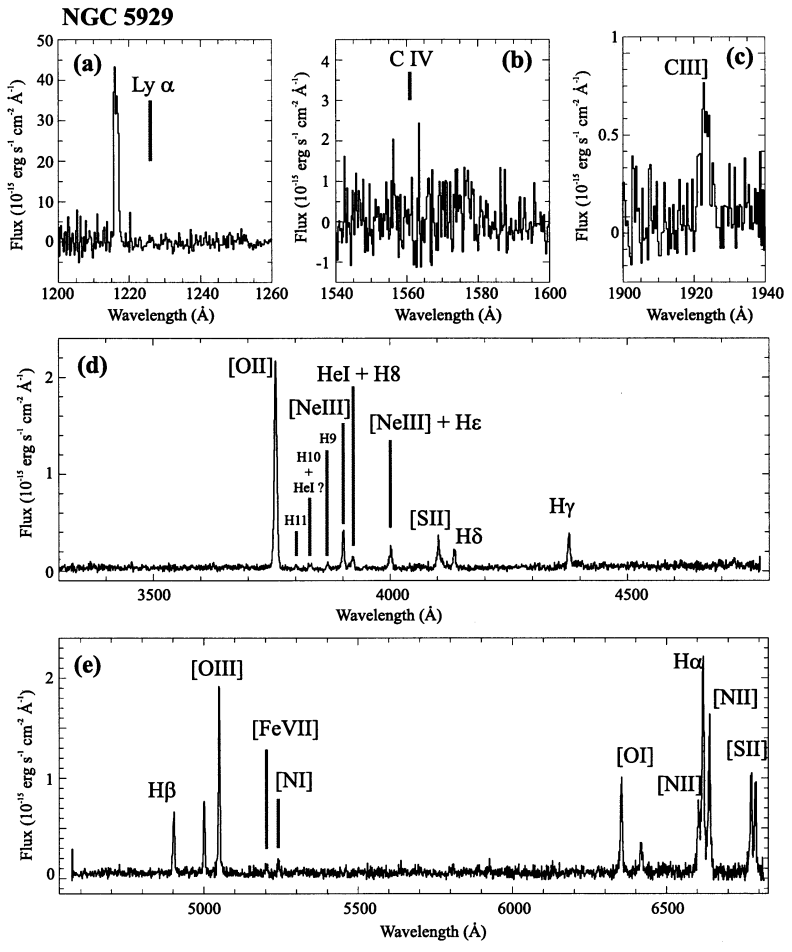

FIG. 4.-HST FOS spectra of NGC 5929. (a) Part of the G130H configuration spectrum where the Ly $\alpha$ emission from the galaxy is expected but not detected. The strong line at $1216 \AA$ is the geocoronal Ly $\alpha \lambda 1215.67$ and the expected location of $\mathrm{Ly} \alpha$ from the galaxy is marked by a vertical line. (b) Part of the $\mathrm{G} 130 \mathrm{H}$ configuration spectrum with the expected location of the C IV $\lambda 1549$ lines marked by a vertical line. (c) Part of the G190H configuration spectrum displaying the $\mathrm{C}$ III] lines. (d) $\mathrm{G} 400 \mathrm{H}$ configuration spectrum. (e) G570H configuration spectrum.

indicates a temperature greater than $16,000 \mathrm{~K}$. For $A_{V}=$ $0.9 \mathrm{mag}$, this temperature increases to the unrealistic value of $48,000 \mathrm{~K}$. If the unusual strength of the [S II] $\lambda \lambda 4069$, 4077 lines is not the result of an instrumental problem in our spectrum, this would suggest that the [S II]-emitting zone is only slightly reddened and that the density is at the high end of our error bars ${ }^{5}\left(540 \mathrm{~cm}^{-3}\right)$. No useful constraint on the $[\mathrm{O} \mathrm{III]}$ and $[\mathrm{N} \mathrm{II}]$ temperatures can be derived from the upper limits on the [O III] $\lambda 4363 /[\mathrm{O}$ III] $\lambda \lambda 4959$, $5007(<23,000 \mathrm{~K})$ and $[\mathrm{N} \mathrm{II}] \lambda 5755 /[\mathrm{N} \mathrm{II}] \lambda \lambda 6548,6583$ $(<30,000 \mathrm{~K})$ ratios.

\subsection{Velocity Profiles and Line Centroids}

The velocity profiles of four major optical emission lines in the $\mathrm{G} 570 \mathrm{H}$ configuration spectra $(\mathrm{H} \beta,[\mathrm{O}$ III $] \lambda 5007$, [N II] $\lambda 6583$, and $\mathrm{H} \alpha$ ) are displayed in Figures 5 (NGC 2110) and 6 (NGC 5929). In both NGC 2110 and NGC 5929 , the velocity profiles have wings extending up to \pm 500 $\mathrm{km} \mathrm{s}^{-1}$, which are stronger than those expected from a Gaussian profile. These wings are also present in the [O II] $\lambda \lambda 3726,3729$ lines ( $\mathrm{G} 400 \mathrm{H}$ configuration).

Comparison of the profiles of the two Balmer lines in NGC 2110 (see Fig. 5, right-hand panel) shows that $\mathrm{H} \alpha$ displays a steeper red side than $\mathrm{H} \beta$. However, as outlined in $\S 3.6$, this side of the $\mathrm{H} \alpha$ line is not very reliably determined, and this uncertainty may contribute to the difference. Apart from a slight blueshift, the [O III] $\lambda 5007$ and [N II] $\lambda 6583$ profiles are very similar to the $\mathrm{H} \beta$ one. These blueshifts of

\footnotetext{
${ }^{5}$ For a given temperature, the $[\mathrm{S}$ II $] \lambda \lambda 4069,4077 /[\mathrm{S}$ II $] \lambda \lambda 6717,6731$ ratio increases with density.
} 
TABLE 4

LINE FLUXES FOR NGC 2110 (see § 3.4)

\begin{tabular}{|c|c|c|c|c|c|}
\hline Line(s) & Flux $^{a}$ & $\begin{array}{c}F / F_{\mathrm{H} \beta}{ }^{\mathbf{b}} \\
\left(A_{V}=0 \mathrm{mag}\right)\end{array}$ & $\begin{array}{c}F / F_{\mathrm{H} \beta}{ }^{\mathrm{c}} \\
\left(A_{V}=1.05 \mathrm{mag}\right)\end{array}$ & $\begin{array}{c}F / F_{\mathrm{H} \beta}{ }^{\mathrm{c}} \\
\left(A_{V}=1.2 \mathrm{mag}\right)\end{array}$ & $\begin{array}{c}F / F_{\mathrm{H} \beta}{ }^{\mathrm{c}} \\
\left(A_{V}=1.4 \mathrm{mag}\right)\end{array}$ \\
\hline (1) & $14 \pm 6$ & $330 \pm 190$ & $3000 \pm 1800$ & $4200 \pm 2500$ & $6400 \pm 3800$ \\
\hline $\mathrm{C}$ IV $\lambda \lambda 1548,1551 \ldots \ldots \ldots \ldots$ & $<2$ & $<60$ & $<220$ & $<270$ & $<360$ \\
\hline C III] $\lambda \lambda 1909,1911 \ldots \ldots \ldots \ldots . .$. & $2.90 \pm 1.0$ & $70 \pm 30$ & $290 \pm 150$ & $350 \pm 180$ & $460 \pm 230$ \\
\hline$[\mathrm{Ne} v] \lambda 3426 \ldots \ldots \ldots \ldots \ldots$ & $0.80 \pm 0.16$ & $19 \pm 7$ & $29 \pm 10$ & $31 \pm 11$ & $33 \pm 12$ \\
\hline$[\mathrm{O}$ II $] \lambda \lambda 3726,3729 \ldots \ldots \ldots \ldots$ & $21.1 \pm 0.3$ & $490 \pm 90$ & $710 \pm 130$ & $750 \pm 130$ & $800 \pm 140$ \\
\hline$[\mathrm{Ne}$ III $] \lambda 3869 \ldots \ldots \ldots \ldots \ldots \ldots$ & $4.2 \pm 0.3$ & $98 \pm 23$ & $140 \pm 30$ & $140 \pm 30$ & $150 \pm 40$ \\
\hline He I $23889+\mathrm{H} 8 \ldots \ldots \ldots \ldots \ldots$ & $0.6 \pm 0.4$ & $14 \pm 12$ & $19 \pm 16$ & $20 \pm 17$ & $22 \pm 18$ \\
\hline$[\mathrm{Ca}$ II $] \lambda 3934 \ldots \ldots \ldots \ldots \ldots \ldots$ & $<0.3$ & $<8$ & $<11$ & $<2$ & $<13$ \\
\hline$[\mathrm{Ne}$ III $] \lambda 3969+\mathrm{H} \epsilon \ldots \ldots \ldots \ldots$ & $1.68 \pm 0.22$ & $39 \pm 11$ & $53 \pm 16$ & $55 \pm 16$ & $58 \pm 17$ \\
\hline$[\mathrm{S}$ II $] \lambda \lambda 4069,4077 \ldots \ldots \ldots \ldots$ & $1.5 \pm 0.3$ & $35 \pm 13$ & $46 \pm 17$ & $47 \pm 17$ & $50 \pm 18$ \\
\hline $\mathrm{H} \delta \ldots \ldots \ldots \ldots \ldots \ldots \ldots \ldots \ldots \ldots \ldots \ldots \ldots \ldots \ldots$ & $0.81 \pm 0.20$ & $19 \pm 8$ & $24 \pm 10$ & $25 \pm 10$ & $27 \pm 11$ \\
\hline 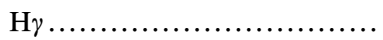 & $1.6 \pm 0.3$ & $37 \pm 13$ & $44 \pm 16$ & $45 \pm 16$ & $47 \pm 16$ \\
\hline$[\mathrm{O}$ III $] \lambda 4363 \ldots \ldots \ldots \ldots \ldots \ldots$ & $0.73 \pm 0.20$ & $17 \pm 7$ & $20 \pm 9$ & $21 \pm 9$ & $21 \pm 9$ \\
\hline He II $\lambda 4686 \ldots \ldots \ldots \ldots \ldots \ldots . . . . . . . .$. & $0.50 \pm 0.28$ & $12 \pm 8$ & $12 \pm 9$ & $12 \pm 9$ & $13 \pm 9$ \\
\hline 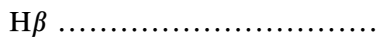 & $4.3 \pm 0.7$ & 100 & 100 & 100 & 100 \\
\hline$[\mathrm{O}$ III $] \lambda 5007 \ldots \ldots \ldots \ldots \ldots \ldots$ & $33.5 \pm 2.9$ & $780 \pm 190$ & $750 \pm 190$ & $740 \pm 190$ & $740 \pm 180$ \\
\hline$[\mathrm{Fe}$ VII $] \lambda 5158+[\mathrm{Fe}$ II $] ? \ldots \ldots$ & $0.4 \pm 0.3$ & $9 \pm 8$ & $9 \pm 8$ & $8 \pm 8$ & $8 \pm 8$ \\
\hline$\left[\mathrm{N}_{\mathrm{I}}\right] \lambda \lambda 5198,5200 \ldots \ldots \ldots \ldots \ldots$ & $1.8 \pm 0.5$ & $42 \pm 18$ & $38 \pm 17$ & $38 \pm 17$ & $37 \pm 16$ \\
\hline$[\mathrm{Fe}$ VII $] \lambda 5276 \ldots \ldots \ldots \ldots \ldots$ & $<0 . \overline{8}$ & $<\overline{22}$ & $<\overline{18}$ & $<\overline{18}$ & $<\overline{17}$ \\
\hline$[\mathrm{N}$ II $] \lambda 5755 \ldots \ldots \ldots \ldots \ldots \ldots \ldots$ & $<0.8$ & $<22$ & $<18$ & $<18$ & $<17$ \\
\hline$[\mathrm{Fe}$ VII $] \lambda 5721 \ldots \ldots \ldots \ldots \ldots$ & $<1$ & $<28$ & $<21$ & $<21$ & $<20$ \\
\hline$\left[\mathrm{O}_{\mathrm{I}}\right] \lambda 6300 \ldots \ldots \ldots \ldots \ldots \ldots$ & $8.2 \pm 1.0$ & $190 \pm 50$ & $140 \pm 40$ & $140 \pm 40$ & $130 \pm 40$ \\
\hline$[\mathrm{S}$ III $] \lambda 6310 \ldots \ldots \ldots \ldots \ldots \ldots$ & $1.0 \pm 0.3$ & $23 \pm 11$ & $17 \pm 8$ & $17 \pm 8$ & $16 \pm 7$ \\
\hline$[\mathrm{Fe} \mathrm{x}] \lambda 6374 \ldots \ldots \ldots \ldots \ldots \ldots$ & $<0.8$ & $<22$ & $<\overline{16}$ & $<\overline{16}$ & $<\overline{15}$ \\
\hline 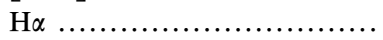 & $18 \pm 4$ & $420 \pm 160$ & $300 \pm 120$ & $290 \pm 110$ & $270 \pm 100$ \\
\hline$[\mathrm{N}$ II $] \lambda 6583 \ldots \ldots \ldots \ldots \ldots \ldots$ & $34 \pm 4$ & $790 \pm 220$ & $570 \pm 160$ & $540 \pm 150$ & $510 \pm 140$ \\
\hline$[\mathrm{S}$ II $] \lambda 6717 \ldots \ldots \ldots \ldots \ldots \ldots$ & $7.9 \pm 0.7$ & $180 \pm 50$ & $130 \pm 30$ & $120 \pm 30$ & $110 \pm 30$ \\
\hline$[\mathrm{S}$ II $] \lambda 6731 \ldots \ldots \ldots \ldots \ldots \ldots$ & $9.8 \pm 0.8$ & $230 \pm 60$ & $160 \pm 40$ & $150 \pm 40$ & $140 \pm 30$ \\
\hline
\end{tabular}

NoTE.-The error bars correspond to a $90 \%$ confidence level.

a Observed line flux in $10^{-15} \mathrm{ergs} \mathrm{s}^{-1} \mathrm{~cm}^{-2}$

${ }^{\text {b }}$ Observed line flux relative to $\mathrm{H} \beta$ with $F_{\mathrm{H} \beta}=100$.

${ }^{c}$ Reddening-corrected line flux relative to $\mathrm{H} \beta$ with $F_{\mathrm{H} \beta}=100$.

the $\left[\mathrm{O} \mathrm{III]}\left(-40 \pm 60 \mathrm{~km} \mathrm{~s}^{-1}\right)\right.$ and $[\mathrm{N} \mathrm{II}](-60 \pm 60 \mathrm{~km}$ $\mathrm{s}^{-1}$ ) lines relative to $\mathrm{H} \beta$ are comparable to the error bars (given as the $90 \%$ confidence level). However, similar blueshifts of a few tens of $\mathrm{km} \mathrm{s}^{-1}$ are observed in the $\mathrm{G} 400 \mathrm{H}$
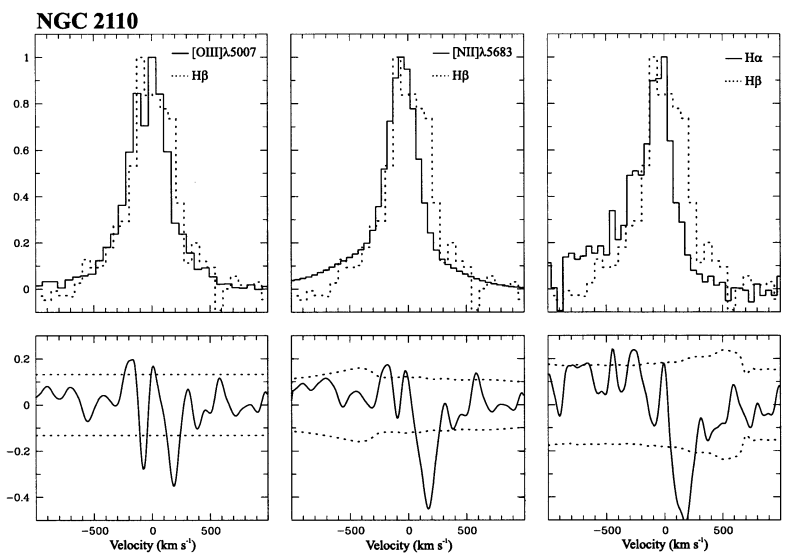

FIG. 5.-Emission-line profiles in NGC 2110. The zero of velocity is arbitrary but is the same for each line, and the peak of each line has been normalized to unity. Left: Velocity profiles (not corrected for instrumental broadening) of the [O III] $\lambda 5007$ (solid line) and $\mathrm{H} \beta$ (dashed line) lines. In the bottom plot, we display the residuals resulting from subtraction of the two velocity profiles ([O III $]-\mathrm{H} \beta$, solid line) as well as the $90 \%$ confidence level (dashed lines) derived from the error analysis described in $\S \S 3.5$ and 3.6. Middle: Same for the [N II] $\lambda 6583$ and $\mathrm{H} \beta$ lines. Right: Same for the $\mathrm{H} \alpha$ and $\mathrm{H} \beta$ lines. spectrum for the [Ne v] $\lambda 3426,[\mathrm{Ne}$ III] $\lambda 3869$, and [O III] $\lambda 4363$ lines with respect to the (weak) Balmer lines. It is notable that much stronger blueshifts have been previously detected in ground-based observations of NGC 2110 (Shuder 1980; $2^{\prime \prime} \times 4^{\prime \prime}$ aperture including our aperture as
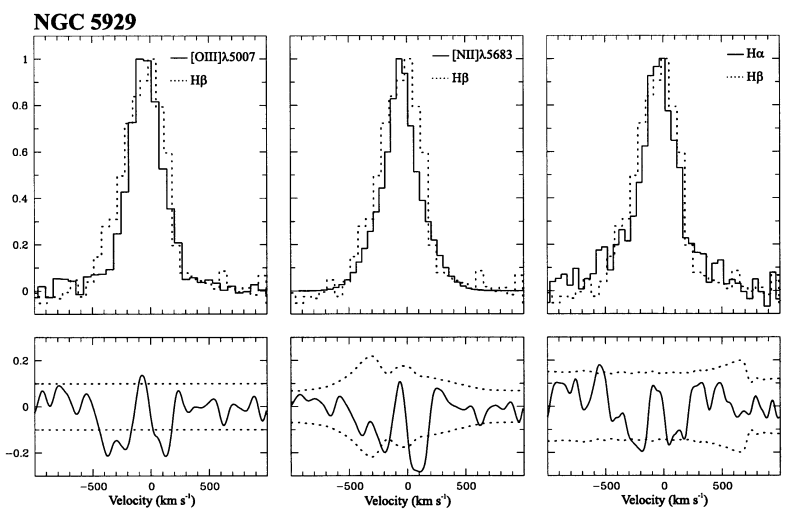

FIG. 6.-Emission-line profiles in NGC 5929. The zero of velocity is arbitrary but is the same for each line, and the peak of each line has been normalized to unity. Left: Velocity profiles (not corrected for instrumental broadening) of the [O III] $\lambda 5007$ (solid line) and $\mathrm{H} \beta$ (dashed line) lines. In the bottom plot, we display the residuals resulting from subtraction of the two velocity profiles ([O III $]-\mathrm{H} \beta$, solid line) as well as the $90 \%$ confidence level (dashed lines) derived from the error analysis described in $\S \S 3.5$ and 3.6. Middle: Same for the [N II] $\lambda 6583$ and $\mathrm{H} \beta$ lines. Right: Same for the $\mathrm{H} \alpha$ and $\mathrm{H} \beta$ lines. 
TABLE 5

LINE FLUXES FOR NGC 5929 (see § 3.4)

\begin{tabular}{|c|c|c|c|c|c|}
\hline Line(s) & Flux $^{a}$ & $\begin{array}{c}F / F_{\mathrm{H} \beta}{ }^{\mathbf{b}} \\
\left(A_{V}=0 \mathrm{mag}\right)\end{array}$ & $\begin{array}{c}F / F_{\mathrm{H} \beta}{ }^{\mathrm{c}} \\
\left(A_{V}=0.6 \mathrm{mag}\right)\end{array}$ & $\begin{array}{c}F / F_{\mathrm{H} \beta}{ }^{\mathrm{c}} \\
\left(A_{V}=0.9 \mathrm{mag}\right)\end{array}$ & $\begin{array}{c}F / F_{\mathrm{H} \beta} \beta^{\mathrm{c}} \\
\left(A_{V}=1.2 \mathrm{mag}\right)\end{array}$ \\
\hline $\operatorname{Ly} \alpha \ldots \ldots \ldots \ldots \ldots \ldots \ldots \ldots \ldots \ldots \ldots \ldots \ldots \ldots \ldots$ & $<6$ & $<160$ & $<580$ & $<1100$ & $<100$ \\
\hline $\mathrm{C}$ iv $\lambda 21548,1551 \ldots \ldots \ldots \ldots$ & $<3$ & $<80$ & $<180$ & $<260$ & $<390$ \\
\hline C III] $\lambda \lambda 1909,1911 \ldots \ldots \ldots \ldots . .$. & $2.2 \pm 0.7$ & $50 \pm 23$ & $110 \pm 50$ & $170 \pm 80$ & $250 \pm 120$ \\
\hline$[\mathrm{Ne} \mathrm{v}] \lambda 3426 \ldots \ldots \ldots \ldots \ldots \ldots$ & $<0.3$ & $<8$ & $<10$ & $<11$ & $<13$ \\
\hline$[\mathrm{O}$ II $] \lambda \lambda 3726,3729 \ldots \ldots \ldots \ldots$ & $14.8 \pm 0.3$ & $340 \pm 50$ & $410 \pm 60$ & $460 \pm 70$ & $510 \pm 80$ \\
\hline$[\mathrm{Ne}$ III $] \lambda 3869 \ldots \ldots \ldots \ldots \ldots \ldots$ & $2.21 \pm 0.26$ & $50 \pm 13$ & $61 \pm 15$ & $67 \pm 17$ & $73 \pm 19$ \\
\hline Не г $23889+$ H8 ........... & $0.81 \pm 0.20$ & $18 \pm 7$ & $22 \pm 8$ & $24 \pm 9$ & $27 \pm 10$ \\
\hline$[\mathrm{Ca}$ II $] \lambda 3934 \ldots \ldots \ldots \ldots \ldots \ldots$ & $<0.2$ & $<5$ & $<6$ & $<7$ & $<8$ \\
\hline$[\mathrm{Ne}$ III $] \lambda 3969+\mathrm{H} \epsilon \ldots \ldots \ldots \ldots$ & $1.6 \pm 0.3$ & $36 \pm 12$ & $43 \pm 14$ & $47 \pm 15$ & $51 \pm 17$ \\
\hline [S II] $\lambda \lambda 4069,4077 \ldots \ldots \ldots \ldots \ldots$ & $2.0 \pm 0.4$ & $45 \pm 15$ & $53 \pm 18$ & $57 \pm 19$ & $62 \pm 21$ \\
\hline $\mathrm{H} \delta \ldots \ldots \ldots \ldots \ldots \ldots \ldots \ldots \ldots \ldots \ldots \ldots \ldots \ldots$ & $1.09 \pm 0.22$ & $25 \pm 8$ & $29 \pm 10$ & $31 \pm 10$ & $33 \pm 11$ \\
\hline 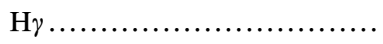 & $1.73 \pm 0.27$ & $39 \pm 11$ & $43 \pm 13$ & $46 \pm 13$ & $48 \pm 14$ \\
\hline$[\mathrm{O}$ III $] \lambda 4363 \ldots \ldots \ldots \ldots \ldots \ldots$ & $<0.4$ & $<10$ & $<12$ & $<12$ & $<\overline{13}$ \\
\hline He II $\lambda 4686 \ldots \ldots \ldots \ldots \ldots \ldots$ & $<0.5$ & $<13$ & $<14$ & $<14$ & $<4$ \\
\hline 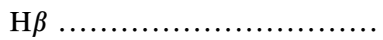 & $4.4 \pm 0.6$ & 100 & 100 & 100 & 100 \\
\hline$[\mathrm{O}$ III $] \lambda 5007 \ldots \ldots \ldots \ldots \ldots \ldots$ & $12.7 \pm 0.9$ & $290 \pm 60$ & $280 \pm 60$ & $280 \pm 60$ & $270 \pm 60$ \\
\hline$[\mathrm{Fe}$ VII $] \lambda 5158+[\mathrm{Fe}$ II $] ? \ldots \ldots$ & $0.5 \pm 0.5$ & $\ldots$ & $\ldots$ & $\ldots$ & $\ldots$ \\
\hline$\left[\mathrm{N}_{\mathrm{I}}\right] \lambda \lambda 5198,5200 \ldots \ldots \ldots \ldots$ & $1.2 \pm 0.5$ & $27 \pm 15$ & $26 \pm 14$ & $25 \pm 14$ & $25 \pm 14$ \\
\hline$[\mathrm{Fe}$ VII $] \lambda 5276 \ldots \ldots \ldots \ldots \ldots \ldots$ & $<0 . \overline{5}$ & $<13$ & $<\overline{12}$ & $<11$ & $<\overline{0}$ \\
\hline$[\mathrm{N}$ II $] \lambda 5755 \ldots \ldots \ldots \ldots \ldots \ldots$ & $<0.7$ & $<18$ & $<16$ & $<15$ & $<15$ \\
\hline He г $\lambda 5876 \ldots \ldots \ldots \ldots \ldots \ldots$ & $<1$ & $<26$ & $<23$ & $<22$ & $<20$ \\
\hline$[\mathrm{Fe}$ VII $] \lambda 5721 \ldots \ldots \ldots \ldots \ldots$ & $<1$ & $<26$ & $<23$ & $<21$ & $<20$ \\
\hline$[\mathrm{O}$ I $] \lambda 6300 \ldots \ldots \ldots \ldots \ldots \ldots$ & $8.2 \pm 1.0$ & $190 \pm 50$ & $160 \pm 40$ & $140 \pm 40$ & $130 \pm 30$ \\
\hline$[\mathrm{S}$ III $] \lambda 6310 \ldots \ldots \ldots \ldots \ldots \ldots$ & $<0.3$ & $<8$ & $<7$ & $<6$ & $<6$ \\
\hline$[\mathrm{Fe} \mathrm{x}] \lambda 6374 \ldots \ldots \ldots \ldots \ldots \ldots$ & $<0.3$ & $<8$ & $<7$ & $<6$ & $<6$ \\
\hline $\mathrm{H} \alpha \ldots \ldots \ldots \ldots \ldots \ldots \ldots \ldots \ldots \ldots \ldots \ldots \ldots \ldots$ & $20.6 \pm 2.5$ & $470 \pm 120$ & $390 \pm 100$ & $350 \pm 90$ & $320 \pm 80$ \\
\hline$[\mathrm{N}$ II $] \lambda 6583 \ldots \ldots \ldots \ldots \ldots$ & $12.1 \pm 1.6$ & $270 \pm 90$ & $230 \pm 70$ & $210 \pm 60$ & $190 \pm 60$ \\
\hline$[\mathrm{S}$ II $] \lambda 6717 \ldots \ldots \ldots \ldots \ldots \ldots$ & $7.6 \pm 0.7$ & $170 \pm 40$ & $140 \pm 30$ & $130 \pm 30$ & $115 \pm 26$ \\
\hline$[\mathrm{S}$ II $] \lambda 6731 \ldots \ldots \ldots \ldots \ldots \ldots$ & $6.7 \pm 0.6$ & $150 \pm 30$ & $120 \pm 30$ & $112 \pm 25$ & $101 \pm 23$ \\
\hline
\end{tabular}

NoTE.-The error bars correspond to a $90 \%$ confidence level.

${ }^{a}$ Observed line flux in $10^{-15} \mathrm{ergs} \mathrm{s}^{-1} \mathrm{~cm}^{-2}$.

b Observed line flux relative to $\mathrm{H} \beta$ with $F_{\mathrm{H} \beta}=100$.

${ }^{c}$ Reddening-corrected line flux relative to $\mathrm{H} \beta$ with $F_{\mathrm{H} \beta}=100$.

well as the nucleus) for high-excitation iron lines $(-280$ $\mathrm{km} \mathrm{s}^{-1}$, [Fe x] $\lambda 6374 ;-110 \mathrm{~km} \mathrm{~s}^{-1}$, [Fe vII] $\lambda 5721$ and $[\mathrm{Fe} \mathrm{VII}]$ 26086) with respect to lower excitation lines.

In NGC 5929, $\mathrm{H} \beta$ and $\mathrm{H} \alpha$ display the same profile (see Fig. 6), both being slightly broader than the [O III] and $[\mathrm{N}$ II] lines. No significant velocity shift from one line to another is seen in this object. This is consistent with the ground-based spectroscopic observations of this off-nuclear knot (southwest component) by Wilson \& $\operatorname{Keel}(1989$, [O I] to [S II]), Whittle et al. (1986, [O III] line), and Ferruit et al. (1997b, [N II] and $\mathrm{H} \alpha$ lines).

\section{NGC 2110: DISCUSSION}

In NGC 2110, our 0"43 (65 pc) diameter FOS aperture includes the brightest parts of the strongly curved nuclear jetlike feature seen in the HST emission-line images of M94 (the "nuclear jet"; see Fig. 1). In the following, we consider two different scenarios for the ionization and excitation of the gas in our aperture. In the first one, the gas is modeled as a mixture of matter-bounded (MB) and ionizationbounded (IB) gaseous components photoionized by a central source (B96; B97), while in the second one we consider the photoionizing ("autoionizing") shock models of DS95 and DS96. Last, we will discuss whether fast shocks can power the extended $0.1-2.4 \mathrm{keV}$ soft X-ray emission seen in the ROSAT high-resolution imager data of NGC 2110 (Weaver et al. 1995).

\subsection{MB-IB Central Source Photoionization Models}

The first possible scenario for the excitation and ionization of the gas is photoionization by a compact central source. In the following, we will use the two-component, solar abundance, central source photoionization models of B96 and B97 to model the observed line ratios. These MB-IB models are preferred to the "classical," single-zone central source photoionization models (see, e.g., Ferland \& Osterbrock 1986) because they solve a number of problems affecting the latter models (see B96 and references therein). To date, four MB-IB models with an extensive set of predicted line ratios have been published: model S (B96, their Table 2) and models L, M, and H (B97, their Table 1). Each model defines a sequence by varying the ratio, $A_{M / I}$, of the solid angle subtended by MB clouds to the solid angle subtended by IB clouds ( $A_{M / I}=0$, IB component only; $A_{M / I}=$ $+\infty$, MB component only). To judge the quality of the match between a model and the data, we have computed, for each line ratio and each model ( $\mathrm{S}, \mathrm{L}, \mathrm{M}$, or $\mathrm{H}$ ), the range of $A_{M / I}$ corresponding to the observed ratio, after reddening correction $\left(A_{V}\right.$ ranging from 1.05 to $1.4 \mathrm{mag}$, see $\left.\S 3.7\right)$ and taking into account the error bar s. For a model to reproduce the data correctly, the derived ranges of $A_{M / I}$ for the different line ratios must all be consistent.

To compute $A_{M / I}$, we have used the following expression, which is derived from equation (3) in B97. It gives, for a specific model, $A_{M / I}$ as a function of the observed ratio of a 
line $A$ to a line $B\left(R_{A / B}\right)$ :

$$
A_{M / I}=\frac{1}{C_{M / I}} \frac{R_{A / B} \mathscr{R}_{B / H \beta}^{\mathrm{IB}}-\mathscr{R}_{A / \mathrm{H} \beta}^{I B}}{\mathscr{R}_{A / H}^{\mathrm{MB}}-R_{A / B}^{\mathrm{MB}} \mathscr{R}_{B / H}^{\mathrm{MB}},}
$$

where $C_{M / I}$ is the ratio of the $\mathrm{H} \beta$ luminosity of the MB component to that of the IB component (constant for a given model; values taken from $\mathrm{B} 96$ and $\mathrm{B} 97$ ), while $\mathscr{R}_{A / \mathrm{H} \beta}^{\mathrm{MB}}$ and $\mathscr{R}_{A / \mathrm{H} \beta}^{\mathrm{IB}}\left(\mathscr{R}_{B / \mathrm{H} \beta}^{\mathrm{MB}}\right.$ and $\left.\mathscr{R}_{B / \mathrm{H} \beta}^{\mathrm{IB}}\right)$ are the theoretical ratios of the line $A(B)$ to $\mathrm{H} \beta$ for the MB and IB components, respectively. The same equation can be used to derive $A_{M / I}$ as a function of the observed ratio of a line $A$ to $\mathrm{H} \beta$, simply by setting $\mathscr{R}_{B / \mathrm{H} \beta}^{\mathrm{MB}}$ and $\mathscr{R}_{B / \mathrm{H} \beta}^{\mathrm{IB}}$ to unity. Observed line ratios outside the range defined by the theoretical ratios for the MB and IB components are inconsistent with the model (and give negative values of $A_{M / I}$ ).

The observed range of line ratios and the ranges of $A_{M / I}$ obtained with this procedure for models $\mathrm{S}, \mathrm{L}, \mathrm{M}$, and $\mathrm{H}$, are listed in Table 6 and displayed in Figure 7. We have chosen to use not only ratios of lines to $\mathrm{H} \beta$ but also ratios between lines of different ionic species of the same element, which are less sensitive to departures from the solar abundances assumed in the MB-IB models.

We first consider the ratios involving the three major oxygen lines ([O I $] \lambda 6300,[\mathrm{O} \mathrm{II}] \lambda 3727$, and $[\mathrm{O} \mathrm{III}] \lambda 5007$; see Table 6). Models L, M, and $\mathrm{H}$ are ruled out as none of them can reach the observed [O $\mathrm{II}] \lambda 3727 / \mathrm{H} \beta$ ratio, and as models $\mathrm{L}$ and $\mathrm{M}$ also fail to reproduce the other observed oxygen line ratios in a consistent way (the ranges of $A_{M / I}$ suggested for each line do not overlap). Model S reproduces correctly [O III] $\lambda 5007 / \mathrm{H} \beta$, [O II] $\lambda 3727 / \mathrm{H} \beta$, and [O II] $\lambda 3727 /\left[\mathrm{O}\right.$ III] $\lambda 5007$ if $A_{M / I}$ is between 0.3 and 0.6. This range of $A_{M / I}$ overlaps with the range derived from the He II $\lambda 4686 / \mathrm{H} \beta$ ratio $(0.05-0.56)$, which is one of the main $A_{M / I^{-}}$ sensitive diagnostics used by B96. However, model $\mathrm{S}$ fails to

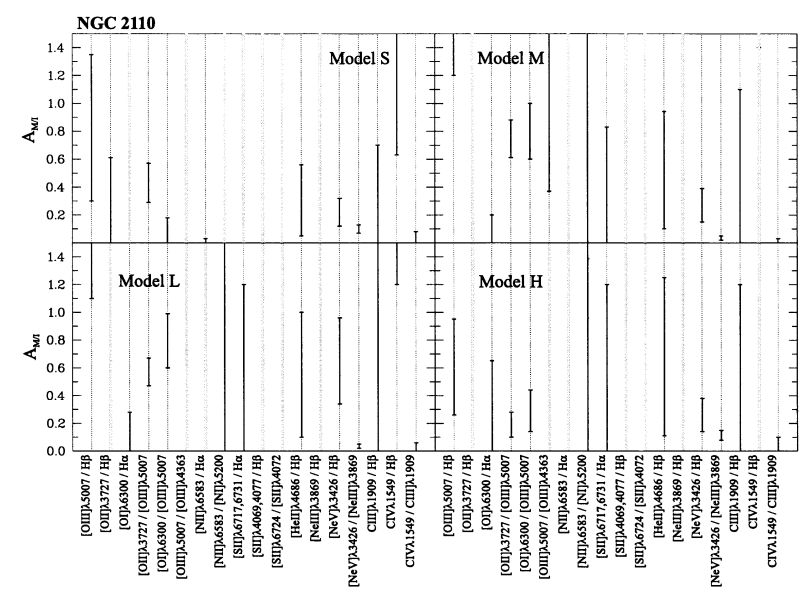

FIG. 7.- Ranges of $A_{M / I}$ obtained using the procedure described in $\S 5.1$, for a set of line ratios observed in NGC 2110, and for the four considered MB-IB models (S, L, M, and H). A gray line is displayed whenever an observed line ratio cannot be reproduced by a model.

reproduce the observed [O I] $\lambda 6300 / \mathrm{H} \alpha$ ratios $(0.31-0.63)$ and, in order to reach the observed [O I] $\lambda 6300 /[\mathrm{O}$ III] $\lambda 5007$ ratio, requires values of $A_{M / I}$ lower than the values inferred from the previous line ratios.

Model S also fails to reproduce the observed [O III] $\lambda 5007 /[\mathrm{O}$ III] $\lambda 4363$ ratio, i.e. the observed [O III] temperature. As shown in Figure 12 of B96, theoretical [O III] temperatures in better agreement with the observed ones could be obtained with model $\mathrm{S}$ using a harder continuum $\left(\alpha=-1.1\right.$ instead of -1.3 for model $\left.\mathrm{S}, F \propto v^{\alpha}\right)$, which would be more consistent with the observed hard X-ray continuum in NGC 2110 (photon index $\Gamma=\alpha-1 \sim-1.5$, with $N \propto v^{\Gamma}$; see, e.g., Hayashi et al. 1996; Weaver et al. 1995). This might increase the fluxes of the low-excitation

TABLE 6

Various Line Ratios Observed in NGC 2110 with Their Corresponding Values of $A_{M / I}$ For Four Published MB-IB MODELS (MODEL S, B96; MODELS L, M AND H, B97)

\begin{tabular}{|c|c|c|c|c|c|}
\hline \multirow[b]{2}{*}{ LINES } & \multirow[b]{2}{*}{ OBSERVED LINE RATIO } & \multicolumn{4}{|c|}{$A_{M / I}$ RANGES } \\
\hline & & Model S & Model L & Model M & Model H \\
\hline$[\mathrm{O}$ III $] \lambda 5007 / \mathrm{H} \beta \ldots \ldots \ldots \ldots \ldots . . .$. & $5.6-9.4$ & $0.30-1.35$ & $1.1-2.4$ & $1.2-2.8$ & $0.26-0.95$ \\
\hline$[\mathrm{O}$ II $] \lambda 3727 / \mathrm{H} \beta \ldots \ldots \ldots \ldots \ldots \ldots \ldots \ldots$ & $5.8-9.4$ & $<0.61$ & $*$ & $*$ & $*$ \\
\hline$[\mathrm{O}$ I $] \lambda 6300 / \mathrm{H} \alpha \ldots \ldots \ldots \ldots \ldots \ldots$ & $0.31-0.63$ & $*$ & $<0.28$ & $<0.20$ & $<0.65$ \\
\hline$[\mathrm{O}$ II $] \lambda 3727 /[\mathrm{O}$ III $] \lambda 5007 \ldots \ldots .$. & $0.85-1.20$ & $0.57-0.29$ & $0.67-0.47$ & $0.88-0.61$ & $0.28-0.10$ \\
\hline$[\mathrm{O}$ I $] \lambda 6300 /[\mathrm{O}$ III $] \lambda 5007 \ldots \ldots \ldots$ & $0.14-0.23$ & $<0.18$ & $0.99-0.60$ & $1.0-0.6$ & $0.44-0.14$ \\
\hline$[\mathrm{O} \mathrm{III}] \lambda 5007 /[\mathrm{O} \mathrm{III}] \lambda 4363 \ldots \ldots$. & $22-50$ & $*$ & $*$ & $>0.37$ & $>1.5$ \\
\hline$[\mathrm{N}$ II $] \lambda 6583 / \mathrm{H} \alpha \ldots \ldots \ldots \ldots \ldots \ldots \ldots$ & $1.3-2.5$ & $<0.03$ & $*$ & $*$ & $*$ \\
\hline$[\mathrm{N}$ II $] \lambda 6583 /[\mathrm{N}$ II $] \lambda 5755 \ldots \ldots \ldots$ & $>32$ & $<4000$ & $<4500$ & $<2300$ & $<8500$ \\
\hline$[\mathrm{N}$ II $] \lambda 6583 /\left[\mathrm{N}_{\mathrm{I}}\right]^{\bar{\lambda}} 5200 \ldots \ldots \ldots . .$. & $8-21$ & $*$ & $<200$ & $<490$ & $<2600$ \\
\hline$[\mathrm{S}$ II $] \lambda \lambda 6717,6731 / \mathrm{H} \alpha \ldots \ldots \ldots \ldots .$. & $0.67-1.25$ & $*$ & $<1.2$ & $<0.83$ & $<1.2$ \\
\hline$[\mathrm{S}$ II $] \lambda \lambda 4069,4077 / \mathrm{H} \beta \ldots \ldots \ldots \ldots . .$. & $0.29-0.68$ & $*$ & $*$ & $*$ & $*$ \\
\hline$[\mathrm{S}$ II $] \lambda 6724 /[\mathrm{S}$ II $] \lambda 4072 \ldots \ldots \ldots . .$. & $3.6-8.1$ & $*$ & $*$ & $*$ & $*$ \\
\hline He II $\lambda 46886 / \mathrm{H} \beta \ldots \ldots \ldots \ldots \ldots \ldots$ & $0.03-0.22$ & $0.05-0.56$ & $0.10-1.0$ & $0.1-0.94$ & $0.11-1.25$ \\
\hline$[\mathrm{Ne} \mathrm{III}] \quad \lambda 3869 / \mathrm{H} \beta \ldots \ldots \ldots \ldots \ldots \ldots$ & $1.1-1.9$ & $*$ & $>32$ & $*$ & $>3.5$ \\
\hline$[\mathrm{Ne} v] \lambda 3426 / \mathrm{H} \beta \quad \ldots \ldots \ldots \ldots \ldots . . .$. & $0.19-0.45$ & $0.12-0.32$ & $0.34-0.96$ & $0.15-0.39$ & $0.14-0.38$ \\
\hline$[\mathrm{Ne} \mathrm{v}] \lambda 3426 /[\mathrm{Ne}$ III $] \lambda 3869 \ldots \ldots$ & $0.15-0.28$ & $0.07-0.13$ & $0.02-0.05$ & $0.02-0.05$ & $0.08-0.15$ \\
\hline $\mathrm{C}$ IV $\lambda 1549 / \mathrm{H} \beta \ldots \ldots \ldots \ldots \ldots \ldots . . . . . .$. & $<3.6$ & $<0.70$ & $<3.2$ & $<1.1$ & $<1.2$ \\
\hline $\mathrm{C}$ III] $\lambda 1909 / \mathrm{H} \beta \ldots \ldots \ldots \ldots \ldots \ldots$ & $1.4-6.9$ & $>0.63$ & $>1.2$ & $>1.4$ & $>5.1$ \\
\hline $\mathrm{C}$ IV $\lambda 1549 / \mathrm{C}$ III] $\lambda 1909 \ldots \ldots \ldots .$. & $<1$ & $<0.08$ & $<0.06$ & $<0.03$ & $<0.1$ \\
\hline
\end{tabular}

Note. $-A_{M / I}$ : ratio of the solid angle subtended by the MB component to the solid angle subtended by the IB component). The ranges of observed line ratios correspond to the allowed range of obscuration $\left(1.05<A_{V}<1.4 \mathrm{mag}\right)$. The symbol * is given when the observed ratio cannot be reproduced by the model. The ratio of the $\mathrm{H} \beta$ luminosity of the MB component to that of the IB component, $C_{M / I}$, is $0.568,0.354,0.368$, and 0.461 for the models $\mathrm{S}, \mathrm{L}, \mathrm{M}$ and $\mathrm{H}$, respectively (B96, B97). 
lines such as $\left[\mathrm{O}_{\mathrm{I}}\right] \lambda 6300$ in the IB component and therefore help to solve the problems with the [ [ $\mathrm{O}$ I] $\lambda 6300$ line strength mentioned above. Model S also underpredicts the observed [N II] $\lambda \lambda 6548,6583 / \mathrm{H} \alpha$ and $[\mathrm{S}$ II] $\lambda \lambda 6717$, $6731 / \mathrm{H} \alpha$ ratios. As for the [O I] $\lambda 6300 / \mathrm{H} \alpha$ ratio, this problem could be at least partially solved if a harder continuum was used in the model. A second possibility is that the IB component extends further than assumed in the models $\left(F_{\mathrm{IB}}=0.97\right.$ in model S; B96), boosting the fluxes of the low excitation lines in the IB component. The values of $A_{M / I}$ derived in model S from the $[\mathrm{Ne} \mathrm{v}] \lambda 3426 / \mathrm{H} \beta$ and $\mathrm{He} \mathrm{II} / \mathrm{H} \beta$ ratios agree well, but the predicted range of $[\mathrm{Ne} \mathrm{III}] \lambda 3869$ / $\mathrm{H} \beta$ is almost 2 times lower than the observed value of 1.11.9 .

In the UV, the only constraint from the observations involves the C IV $\lambda 1549$ and C III] $\lambda 1909$ emission lines. For models $\mathrm{M}$ and $\mathrm{H}$, the two reddening-sensitive $\mathrm{C}$ IV $\lambda 1549$ / $\mathrm{H} \beta$ and $\mathrm{C} \mathrm{III]} \lambda 1909 / \mathrm{H} \beta$ ratios indicate very different ranges of $A_{M / I}$ and cannot be reproduced simultaneously. The values of $A_{M / I}$ from these ratios are consistent for models $\mathrm{S}$ and L, but only for model S do we obtain a range of $A_{M / I}$ values $\left(0.63<A_{M / I}<0.70\right)$ reasonably close to the values suggested by the optical line ratios. However, even in the case of model S, we cannot reproduce correctly the C IV $\lambda 1549 / \mathrm{C} \mathrm{III]} \lambda 1909$ ratio.

Therefore, none of the four published MB-IB models successfully reproduce all the observed line ratios. Although model $\mathbf{S}$ is in better agreement with the data than models $\mathrm{L}$, $\mathbf{M}$, and $\mathrm{H}$, it still provides only a poor match to the observations. However, it is likely that fine-tuning of model parameters, such as the hardness of the ionizing continuum, the abundances, or the truncation threshold of the IB component, would significantly improve the agreement.

\subsection{Photoionizing Shock Models}

The other candidate for the excitation and ionization of the gas in our aperture is fast shock waves, presumably generated by the propagation of the radio ejecta (Ulvestad \& Wilson 1984; Nagar et al. 1999) into the ISM of the host galaxy. The only available kinematical and spectral evi- dence for shocks in the inner regions comes from the nearinfrared spectroscopic observations of Storchi-Bergmann et al. (1999). They found differences between the kinematics of the gas emitting [ $\mathrm{Fe}$ II $]$ and that emitting $\mathrm{Pa} \beta$ and $\mathrm{H}_{2}$, and a broadening of the $[\mathrm{Fe}$ II] profile at the locations where the $[\mathrm{Fe}$ II $] \lambda 1.257 \mu \mathrm{m} / \mathrm{Pa} \beta$ ratio is higher.

To simplify the comparison of central source photoionization and shock models, we will restrict ourselves to shock models in which the influence of the compact central source of ionizing radiation on the gas is negligible. Models describing the spectral properties of shocks (with or without the precursor included) have been developed and applied to active galaxies by various authors (Contini \& Aldrovandi 1983; Aldrovandi \& Contini 1985; Viegas-Aldrovandi \& Contini 1989; Contini 1997; Binette, Dopita, \& Tuohy 1985; DS95; DS96). In the following, we will consider only the recent shock + precursor models of DS95 and DS96 with solar abundances, which have the advantage of accounting for the line emission of both the shock region and the photoionized precursor.

\subsubsection{Line Ratios}

In Table 7, we list various line ratios with the range of reddening corrected values inferred from the observations (for $A_{V}$ in the range 1.05-1.4 mag), and the values predicted by the shock + precursor models (as derived from Tables 8-11 in DS96) for four shock velocities $\left(V_{S}=200\right.$, 300,400 , and $500 \mathrm{~km} \mathrm{~s}^{-1}$ ). The range predicted by the models corresponds to a range of magnetic parameters $B_{0} / n_{0}^{1 / 2}$ between 0 and $4 \mu \mathrm{G} \mathrm{cm}{ }^{3 / 2}\left(B_{0}\right.$ is the perpendicular component of the preshock magnetic field and $n_{0}$ is the preshock density). Shock models without precursors (i.e., with only the shock region) are ruled out by the observed [O II $] \lambda 3727 /[\mathrm{O} \mathrm{III}] \lambda 5007$ ratio $(0.85-1.20)$, which is clearly in the shock + precursor domain of Figure 1 in DS95, and by the relatively high observed [O III] $\lambda 5007 / \mathrm{H} \beta$ ratio (5.69.4), which cannot be reproduced without the contribution of the precursor.

A comparison between the observed and predicted values listed in Table 7 for the major optical oxygen emission lines ([O I] $\lambda 6300,[\mathrm{O}$ II $] \lambda 3727$, and [O III] $\lambda 5007$ ), rules out

TABLE 7

LINE RATIOS IN NGC 2110

\begin{tabular}{|c|c|c|c|c|c|}
\hline Line Ratio & Observed & $V_{S}=200 \mathrm{~km} \mathrm{~s}^{-1}$ & $V_{S}=300 \mathrm{~km} \mathrm{~s}^{-1}$ & $V_{S}=400 \mathrm{~km} \mathrm{~s}^{-1}$ & $V_{S}=500 \mathrm{~km} \mathrm{~s}^{-1}$ \\
\hline$[\mathrm{O}$ III] $\lambda 5007 / \mathrm{H} \beta \ldots \ldots \ldots \ldots \ldots$ & $5.6-9.4$ & $1.52-1.58$ & $5.9-6.5$ & $8.0-9.1$ & $12.5-13.8$ \\
\hline$[\mathrm{O}$ II $] \lambda 3727 / \mathrm{H} \beta \ldots \ldots \ldots \ldots \ldots \ldots$ & $5.8-9.4$ & $2.3-3.6$ & $1.7-5.2$ & $1.7-7.0$ & $1.7-7.2$ \\
\hline$[\mathrm{O}$ I $] \lambda 6300 / \mathrm{H} \alpha \ldots \ldots \ldots \ldots \ldots \ldots$ & $0.31-0.63$ & $0.07-0.13$ & $0.32-0.47$ & $0.57-0.81$ & $0.81-0.95$ \\
\hline$[\mathrm{O}$ II $] \lambda 3727 /[\mathrm{O}$ III $] \lambda 5007 \ldots \ldots .$. & $0.85-1.20$ & $1.5-2.3$ & $0.3-0.8$ & $0.2-0.8$ & $0.1-0.5$ \\
\hline$[\mathrm{O}$ I $] \lambda 6300 /[\mathrm{O}$ III $] \lambda 5007 \ldots \ldots \ldots$ & $0.14-0.23$ & $0.14-0.26$ & $0.15-0.23$ & $0.18-0.30$ & $0.17-0.21$ \\
\hline$[\mathrm{O}$ III $] \lambda 5007 /[\mathrm{O}$ III $] \lambda 4363 \ldots \ldots$. & $22-50$ & $\sim 28$ & $103-108$ & $107-109$ & $79-82$ \\
\hline$[\mathrm{N} \mathrm{II}] \lambda 6583 / \mathrm{H} \alpha \ldots \ldots \ldots \ldots \ldots \ldots . . . .$. & $1.3-2.5$ & $0.19-0.41$ & $0.23-0.65$ & $0.34-0.86$ & $0.39-0.85$ \\
\hline$[\mathrm{S}$ II $] \lambda \lambda 6717,6731 / \mathrm{H} \alpha \ldots \ldots \ldots .$. & $0.67-1.25$ & $0.18-0.32$ & $0.41-0.56$ & $0.48-0.75$ & $0.49-0.78$ \\
\hline He II $\lambda 4686 / \mathrm{H} \beta \ldots \ldots \ldots \ldots \ldots \ldots$ & $0.03-0.22$ & $\sim 0.05$ & $0.23-0.28$ & $0.28-0.37$ & $0.23-0.31$ \\
\hline$[\mathrm{Ne}$ III] $] \lambda 3869 / \mathrm{H} \beta \ldots \ldots \ldots \ldots \ldots$ & $1.1-1.9$ & $0.18-0.21$ & $0.50-0.72$ & $0.71-1.1$ & $1.2-1.6$ \\
\hline$[\mathrm{Ne} \mathrm{v}] \lambda 3426 / \mathrm{H} \beta \ldots \ldots \ldots \ldots \ldots$ & $0.19-0.45$ & $\sim 0.13$ & $\sim 0.10$ & $\sim 0.12$ & $\sim 0.4$ \\
\hline$[\mathrm{Ne} \mathrm{v}] \lambda 3426 /[\mathrm{Ne}$ III $] \lambda 3869 \ldots \ldots$ & $0.15-0.28$ & $0.64-0.73$ & $0.14-0.21$ & $0.11-0.18$ & $0.25-0.37$ \\
\hline $\mathrm{C}$ IV $\lambda 1549 / \mathrm{H} \beta \ldots \ldots \ldots \ldots \ldots \ldots$ & $<3.6$ & $\sim 2.1$ & $1.0-1.1$ & $1.1-1.2$ & $3.3-3.7$ \\
\hline $\mathrm{C}$ III] $\lambda 1909 / \mathrm{H} \beta \ldots \ldots \ldots \ldots \ldots . . . . .$. & $1.4-6.9$ & $0.33-0.38$ & $0.22-0.34$ & $0.49-0.77$ & $1.1-1.4$ \\
\hline $\mathrm{C}$ IV $\lambda 1549 / \mathrm{C}$ III $] \lambda 1909 \ldots \ldots \ldots \ldots$ & $<1$ & $5.6-6.3$ & $3.1-4.9$ & $1.5-2.4$ & $2.5-3.3$ \\
\hline
\end{tabular}

NoTE.-The second column gives the observed range (allowing for the uncertainty in obscuration $-A_{V}=1.05-1.4$ mag), while the third, fourth, fifth, and sixth columns give the values predicted by the photoionizing shock models of DS95 and DS96 (shock + precursor) for four shock velocities, $V_{S}=200,300,400$ and $500 \mathrm{~km} \mathrm{~s}^{-1}$. The range given corresponds to a range of "magnetic parameters," $B / n_{0}^{1 / 2}$, from 0 to $4 \mu \mathrm{G} \mathrm{cm}^{3 / 2}$. 
"low"-velocity shocks $\left(V_{S} \sim 200 \mathrm{~km} \mathrm{~s}^{-1}\right)$, which cannot reproduce the strengths of [O II] $\lambda 3727$ and [O III] $\lambda 5007$. Shock velocities between 300 and $500 \mathrm{~km} \mathrm{~s}^{-1}$ give oxygen line ratios relative to $\mathrm{H} \beta$ and $\mathrm{H} \alpha$ (as well as the [O $\mathrm{I}]$ $\lambda 6300 /[\mathrm{O} \mathrm{III}] \lambda 5007$ ratio) comparable to the observed ones, but the match is poorer for [O II] $\lambda 3727 /[\mathrm{O} \mathrm{III}] \lambda 5007$. The models with $V_{S}=300-500 \mathrm{~km} \mathrm{~s}^{-1}$ also fail to reproduce the [O III] $\lambda 5007 /[\mathrm{O} \mathrm{III}] \lambda 4363$ ratio, which requires a slow shock $\left(V_{S}<300 \mathrm{~km} \mathrm{~s}^{-1}\right)$ so that the majority of the [O III] emission is produced in the high-temperature environment of the postshock region, rather than in the photoionized precursor, which has a temperature of $\simeq 10,000-12,000 \mathrm{~K}$. Although the fast-shock models have been computed in the low-density limit, this discrepancy between the observed and predicted [O III] temperatures is unlikely to be a collisonal deexcitation effect, since the [S II]-inferred density is only $1200_{-300}^{+500} \mathrm{~cm}^{-3}$. Indeed, for a typical $400 \mathrm{~km} \mathrm{~s}^{-1}$ shock velocity, the required preshock density is $<34 \mathrm{~cm}^{-3}$ (see § 5.2.2), too low for significant collisonal deexcitation of the $\left[\mathrm{O} \mathrm{III]} \lambda 5007\right.$ line (critical density of $\simeq 8 \times 10^{5} \mathrm{~cm}^{-3}$ ) to occur in either the precursor or postshock zone.

The strengths of the [Ne III] $\lambda 3869$ and [Ne v] $\lambda 3426$ lines relative to $\mathrm{H} \beta$ indicate shock velocities between 400 and $500 \mathrm{~km} \mathrm{~s}^{-1}$, in agreement with what is suggested by the oxygen lines. On the other hand, the observed He II $\lambda 4686 /$ $\mathrm{H} \beta$ ratio is lower than predicted and suggests shock velocities lower than $300 \mathrm{~km} \mathrm{~s}^{-1}$. Last, the strength of the [S II] $\lambda \lambda 6717,6731$ doublet relative to $\mathrm{H} \alpha$ is correctly predicted for shock velocities between 400 and $500 \mathrm{~km} \mathrm{~s}^{-1}$ (although the predicted range lies at the bottom of the error bars of the $[\mathrm{S} \mathrm{II}] / \mathrm{H} \alpha$ ratio), whereas the observed $[\mathrm{N}$ II $] \lambda 6583 / \mathrm{H} \alpha$ ratio is too high by a factor $2-3$. This discrepancy between the predicted and observed $[\mathrm{N} \mathrm{II]} / \mathrm{H} \alpha$ ratios seems to be systematic (see Fig. $2 b$ in DS95) and could reflect nitrogen abundances higher than solar (DS95). However, an overabundance of nitrogen should induce a weakening of the lines of the other low-excitation species (DS95), which is not seen for [S II] (see above).

Unfortunately, because of the uncertainties in the reddening (\$3.7), the utility of the ultraviolet carbon lines in discriminating between shock-induced and central source photoionization models (as emphasized by Allen et al. 1998), is less than hoped for. No significant constraint on the photoionizing shock models is obtained from the observed upper limit on the $\mathrm{C}$ IV $\lambda 1549 / \mathrm{H} \beta$ ratio $(<3.6)$. The predicted C III] $\lambda 1909 / \mathrm{H} \beta$ ratio is lower than observed for all the shock velocities considered in Table 7. This, together with the fact that the predicted C IV $\lambda 1549 / \mathrm{C}$ III] $\lambda 1909$ ratio is too high compared to the observed upper limit, indicates an observed excess of $\mathrm{C}$ III] $\lambda 1909$ relative to what is expected from the photoionizing shock models. However, this could be a consequence of an overestimate of the extinction at the wavelengths of the $\mathrm{C}$ III] lines, since the extinction curve we have used (Cardelli et al. 1989) includes the $2175 \AA$ UV bump (see Fig. 4 in Cardelli et al. 1989), the wings of which affect the $\mathrm{C}$ III] $\lambda 1909$ lines.

\subsubsection{Preshock Density and Emitting Volume}

The previous section shows that shocks with a velocity of $V_{S} \simeq 400 \mathrm{~km} \mathrm{~s}^{-1}$ can approximately reproduce the observed line ratios. We can use this value of $V_{S}$ and the observed $\mathrm{H} \beta$ luminosity and gas density to estimate the preshock density $\left(n_{0}\right)$, as well as the shock surface area $\left(\mathscr{S}_{\text {shock }}\right)$ and volume $\left(\mathscr{V}_{s+p}\right.$, volume of the shock + precursor structure) required to power the line emission in our aperture.

Using the results in DS96, we have computed the compression factors for a $400 \mathrm{~km} \mathrm{~s}^{-1}$ shock appropriate to the [S II]-emitting zone, which factors are 50,90,160, and 1000 for magnetic parameters $B_{0} / n_{0}^{1 / 2}$ of $4,2,1$, and $0 \mu \mathrm{G} \mathrm{cm}^{3 / 2}$, respectively. Given the density inferred from the observed $[\mathrm{S}$ II $]$ ratio $\left(1200_{-300}^{+500} \mathrm{~cm}^{-3}\right)$, this yields preshock densities in the range $n_{0}=0.9-34 \mathrm{~cm}^{-3}$. From equations (2.2) and (2.4) in DS95 and for a $400 \mathrm{~km} \mathrm{~s}^{-1}$ shock, we can express $\mathscr{S}_{\text {shock }}$ in terms of the absolute $\mathrm{H} \beta$ luminosity, $\mathscr{L}_{\mathrm{H} \beta}$, and the preshock density, $n_{0}$ :

$$
\mathscr{S}_{\text {shock }}\left[\mathrm{cm}^{2}\right]=\frac{1}{4.42 \times 10^{-4}} \frac{\mathscr{L}_{\mathrm{H} \beta}\left[\mathrm{ergs}^{-1}\right]}{n_{0}\left[\mathrm{~cm}^{-3}\right]} .
$$

The absolute $\mathrm{H} \beta$ luminosity in our aperture can be derived from the reddening corrected $\mathrm{H} \beta$ flux, which for $A_{V}=$ $1.05-1.4 \mathrm{mag}$, is in the range 11-22 $\times 10^{-15} \mathrm{ergs} \mathrm{s}^{-1} \mathrm{~cm}^{-2}$. For a distance to NGC 2110 of $32 \mathrm{Mpc}$, this yields $\mathscr{L}_{\mathrm{H} \beta}=$ $1.4-2.7 \times 10^{39} \mathrm{ergs} \mathrm{s}^{-1}$. Using this, together with our estimate of the preshock density, we infer the required shock surface area between $10^{-2}$ and $0.7 \mathrm{kpc}^{2}$, i.e., between 100 $\mathrm{pc} \times 100 \mathrm{pc}\left(0^{\prime \prime} .7 \times 00^{\prime \prime} 7\right)$ and $845 \mathrm{pc} \times 845 \mathrm{pc}\left(5^{\prime \prime} 6 \times 5^{\prime \prime} .6\right)$.

We can also derive a lower limit on the total volume of the shock + precursor association, $\mathscr{V}_{s+p}=\mathscr{S}_{\text {shock }} L_{s+p}$ $\left(L_{s+p}\right.$ being the thickness of the shock + precursor structure). A lower limit on $L_{s+p}$ is given by the thickness of the precursor $\mathrm{H}$ II region $\left(L_{p}\right)$, which is, for a $400 \mathrm{~km} \mathrm{~s}^{-1}$ shock, $L_{s+p}>L_{p}=611\left(n_{0}\left[\mathrm{~cm}^{-3}\right]\right)^{-1}$ pc (eq. [4.3] in DS96). Using this expression, together with equation (3), we derive

$$
\mathscr{V}_{s+p}\left[\mathrm{pc}^{3}\right]=1.46 \times 10^{-31} \frac{\mathscr{L}_{\mathrm{H} \beta}\left[\mathrm{ergs} \mathrm{s}^{-1}\right]}{\left(n_{0}\left[\mathrm{~cm}^{-3}\right]\right)^{2}} .
$$

For our minimum and maximum estimated values of the preshock density, this yields $\mathscr{V}_{s+p}>0.2-0.5 \times 10^{9} \mathrm{pc}^{3}$ $\left(n_{0}=0.9 \mathrm{~cm}^{-3}\right)$ and $\mathscr{V}_{s+p}>0.2-0.4 \times 10^{6} \mathrm{pc}^{3} \quad\left(n_{0}=34\right.$ $\mathrm{cm}^{-3}$ ). Assuming that the ionized gas region in our aperture has a thickness typical of that of a galaxy gas disk $(\simeq 300 \mathrm{pc}$, from H I observations; Dickey \& Lockman 1990), the volume included in our aperture is only $\simeq 10^{6} \mathrm{pc}^{3}$. Therefore, the actual preshock density must be near the high end of our estimates, the compression factor small and the magnetic parameter near the high end of the considered range. This conclusion holds independent of whether the gas is relatively homogeneous or in many clouds.

\subsubsection{Can Fast Shocks Power the Extended X-Ray Emission in NGC 2110 ?}

An interesting issue is whether the fast shocks that may power the extended optical line emission in NGC 2110, as discussed above, can also be responsible for the extended soft X-ray emission discovered $\simeq 4$ " north of the nucleus by Weaver et al. (1995) in their ROSAT High Resolution Imager observations. The $0.1-2.4 \mathrm{keV}$ flux of this extended component is $\simeq 3 \times 10^{-13}$ ergs s${ }^{-1} \mathrm{~cm}^{-2}$ (Weaver et al. 1995; Hayashi et al. 1996), while the total [O III] $\lambda 5007$ fluxes of the "nuclear jet" and "S-shaped" structures are 7.1 and $5.1 \times 10^{-14} \mathrm{ergs} \mathrm{s}^{-1} \mathrm{~cm}^{-2}$, respectively (Table 2 in M94, and assuming a [O III] $\lambda 5007 /[\mathrm{O}$ III] $\lambda 4959$ ratio of 2.88).

For a given shock velocity $\left(V_{S}\right.$ in $\left.\mathrm{km} \mathrm{s}^{-1}\right)$ and an equivalent hydrogen column to the hot gas $\left(N_{\mathrm{H}}\right.$, in $\left.\mathrm{cm}^{-2}\right)$, the predicted 0.1-2.4 keV flux at Earth, $F_{\mathrm{X}}\left(V_{\mathrm{S}}, N_{\mathrm{H}}\right)\left(\right.$ in ergs s ${ }^{-1}$ 
$\mathrm{cm}^{-2}$ ), can be expressed as a function of the observed [O III] $\lambda 5007$ flux, $F_{[\mathrm{O} \text { II] }}$ (in ergs $\mathrm{s}^{-1} \mathrm{~cm}^{-2}$ ), using the relation $F_{\mathrm{X}}\left(V_{S}, N_{\mathrm{H}}\right)=\Gamma\left(V_{S}, N_{\mathrm{H}}\right) F_{\text {[O } \mathrm{m]}}$, with

$$
\Gamma\left(V_{S}, N_{\mathrm{H}}\right)=\frac{\int_{0.1 \mathrm{keV}}^{2.4 \mathrm{keV}} \tau_{\mathrm{X}}\left(E, N_{\mathrm{H}}\right) \Phi_{X}^{0}\left(E, V_{S}, n_{0}\right) d E}{\tau \mathscr{R}_{[\mathrm{O} \text { II] }] / \mathrm{H} \beta}\left(V_{S} S\right) \Phi_{\mathrm{H} \beta}^{0}\left(V_{S}, n_{0}\right)},
$$

where $\tau_{\mathrm{X}}\left(E, N_{\mathrm{H}}\right)$ accounts for photoelectric absorption of the observed X-rays; $\Phi_{\mathrm{X}}^{0}\left(E, V_{S}, n_{0}\right)$ is the theoretical 0.1-2.4 $\mathrm{keV}$ flux per unit area of shock surface at the energy $E$ (in ergs $\mathrm{s}^{-1} \mathrm{~cm}^{-2} \mathrm{keV}^{-1}$ ) in the absence of absorption; $\Phi_{\mathrm{H} \beta}^{0}\left(V_{S}, n_{0}\right)$ is the theoretical $\mathrm{H} \beta$ flux per unit area of shock surface (in ergs $\mathrm{s}^{-1} \mathrm{~cm}^{-2}$ ) in the absence of extinction; $\mathscr{R}_{[\mathrm{O} \text { III]/H } \beta}\left(V_{S}\right)$ is the theoretical [O III] $\lambda 5007 / \mathrm{H} \beta$ ratio for a $V_{S} \mathrm{~km} \mathrm{~s}^{-1}$ photoionizing shock (this ratio depends only slightly on the magnetic parameter and very marginally on the preshock density); and $\tau=0.32$ is the extinction coefficient at $5007 \AA$ for the value $A_{V}=1.05 \mathrm{mag}$ inferred from the observations (extinction curve from Cardelli et al. 1989). The key point is that the dependence of both $\Phi_{\mathrm{X}}^{0}\left(E, V_{S}, n_{0}\right)$ and $\Phi_{\mathrm{H} \beta}^{0}\left(V_{S}, n_{0}\right)$ on $n_{0}$ is the same, so their ratio, and therefore $\Gamma$, are independent of $n_{0}$, an unknown quantity (cf. Wilson \& Raymond 1999). $\Phi_{\mathrm{X}}^{0}\left(E, V_{S}, n_{0}\right), \Phi_{\mathrm{H} \beta}^{0}\left(V_{S}, n_{0}\right)$ and $\mathscr{R}_{[\mathrm{O} \text { II] } / \mathrm{H} \beta}\left(V_{S}\right)$ can be obtained directly from the photoionizing shock models using Table 4 of DS96, equations (2.2) and (2.4) of DS95, and Tables 8-11 of DS96, respectively.

The theoretical, photoelectrically absorbed $0.1-2.4 \mathrm{keV}$ X-ray flux per unit of shock surface, $\Phi_{\mathrm{X}}\left(V_{S}, n_{0}\right)=$ $\int \tau_{\mathrm{X}} \Phi_{\mathrm{X}}^{0} d E$, was calculated as follows. First, the $5 \mathrm{eV}-11 \mathrm{keV}$ unabsorbed fluxes of photoionizing shocks with velocities between 200 and $500 \mathrm{~km} \mathrm{~s}^{-1}$ (Table 4 in DS96 $6^{6}$; electronic version in Leitherer et al. 1996) have been appropriately formatted to be used as a model in the XSPEC software (Arnaud 1996). Then, for each shock velocity between 200 and $500 \mathrm{~km} \mathrm{~s}^{-1}$, we have used XSPEC to compute the photoelectrically absorbed spectra of the photoionizing shocks, assuming an equivalent hydrogen absorbing column equal to the Galactic hydrogen column density toward NGC 2110 of $N_{\mathrm{H}}=0.186 \times 10^{22} \mathrm{~cm}^{-2}$ (Weaver et al. 1995; Hayashi et al. 1996). For a normal gas-to-dust ratio, this column corresponds to $A_{V}=1.0 \mathrm{mag}$, quite close to the values inferred from the Balmer decrement (see $\S 3.7$ ). Last, the resulting spectra were integrated between 0.1 and $2.4 \mathrm{keV}$ to give $\Phi_{\mathrm{X}}\left(V_{S}, n_{0}\right)$.

The resulting values of $\Phi_{\mathrm{X}}\left(V_{S}, n_{0}\right), \quad \Phi_{\mathrm{H} \beta}^{0}\left(V_{S}, n_{0}\right)$, $\mathscr{R}_{[\mathrm{O} \text { II] } / \mathrm{H} \beta}\left(V_{S}\right)$, and $\Gamma\left(V_{S}, N_{\mathrm{H}}\right)$ for shock velocities of 200,300 ,

\footnotetext{
${ }^{6}$ The photon fluxes in this Table 4 of DS96 are per steradian and not integrated over $4 \pi$ as quoted.
}

400 , and $500 \mathrm{~km} \mathrm{~s}^{-1}$ and the above value of $N_{\mathrm{H}}$, are tabulated in Table 8. The extremely large increase of $\Phi_{\mathrm{X}}$ from 200 to $300 \mathrm{~km} \mathrm{~s}^{-1}$ is a result of the combined effect of the steep increase in the total energy radiated in X-rays and the decrease in the effective photoelectric absorption as relatively more of the X-rays are emitted at higher energies (see Fig. 2 in DS96).

The expected 0.1-2.4 keV fluxes for the "nuclear jet" and "S-shaped" emission-line features in NGC 2110, based on their observed [O III] $\lambda 5007$ fluxes, are given in the bottom rows of Table 8 . It is found that only shocks with velocities $\geq 500 \mathrm{~km} \mathrm{~s}^{-1}$ are capable of accounting for the observed $0.1-2.4 \mathrm{keV}$ flux of the extended X-rays $\left(\simeq 3.0 \times 10^{-13} \mathrm{ergs}\right.$ $\mathrm{s}^{-1} \mathrm{~cm}^{-2}$ ). Shocks of lower velocities are far too soft and weak sources of X-rays.

It is then of interest to see if the emission-line profiles are consistent with the presence of such $500 \mathrm{~km} \mathrm{~s}^{-1}$ shocks at the location of the extended X-ray emission $(\simeq 4$ " $[600 \mathrm{pc}]$ north of the nucleus). The $\mathrm{H} \beta$ and [O III] $\lambda 5007$ line profiles $4^{\prime \prime}[600 \mathrm{pc}]$ north of the nucleus are similar and relatively narrow ( $\simeq 200 \mathrm{~km} \mathrm{~s}^{-1}$; Fig. 10 in Wilson et al. 1985). In particular, the broadening of $\mathrm{H} \beta$ relative to [O III] $\lambda 5007$ expected in the photoionizing shock models (most of the [O III] is emitted in the quiescent precursor, while roughly half of the $\mathrm{H} \beta$ comes from the kinematically disturbed shock region; DS96) is not observed. The same problem is present in the near-infrared, where the $[\mathrm{Fe}$ II] lines, which may be emitted in material accelerated by the radio jet (Storchi-Bergmann et al. 1999), have a FWHM of only $240 \pm 40 \mathrm{~km} \mathrm{~s}^{-1}, 2.9$ [435 pc] north of the nucleus (Storchi-Bergmann et al. 1999).

Alternatively, the hot, X-ray-emitting gas could be created in shocks closer to the nucleus, e.g., in the "nuclear jet," and could then expand out to $\simeq 4$ " $[600 \mathrm{pc}]$. Indeed, the regions closer to the nucleus display much broader line profiles, consistent with the presence of shocks with velocities $\geq 500 \mathrm{~km} \mathrm{~s}^{-1}$ : there are (1) line profiles with wings extending up to $\pm 500 \mathrm{~km} \mathrm{~s}^{-1}$ in our FOS aperture located 0.5 from the nucleus (see $\S 4.3$ ), (2) $\mathrm{H} \beta$ and [O III] profiles with $\sim 500 \mathrm{~km} \mathrm{~s}^{-1}$ FWHM in ground-based observations (Wilson et al. 1985), and (3) $[\mathrm{Fe} \mathrm{II}]$ and $\mathrm{Pa} \beta$ nuclear line profiles exhibiting very broad wings (especially $\mathrm{Pa} \beta$; see Fig. 4, upper right panel, in Storchi-Bergmann et al. 1999). However, this scenario has two main problems. First, the line ratios observed in the "nuclear jet" indicate shock velocities lower than $500 \mathrm{~km} \mathrm{~s}^{-1}$ (see $\S$ 5.2.1). Second, the X-ray observations show that most of the excess 0.1-2.4

TABLE 8

PRedicted 0.1-2.4 keV FluXes

\begin{tabular}{|c|c|c|c|c|c|}
\hline Parameter & & $V_{S}=200 \mathrm{~km} \mathrm{~s}^{-1}$ & $V_{S}=300 \mathrm{~km} \mathrm{~s}^{-1}$ & $V_{S}=400 \mathrm{~km} \mathrm{~s}^{-1}$ & $V_{S}=500 \mathrm{~km} \mathrm{~s}^{-1}$ \\
\hline$\Phi_{X}\left(V_{S}, n_{0}\right)\left(\mathrm{ergs} \mathrm{s}^{-1} \mathrm{~cm}^{-2}\right) \ldots \ldots$ & & $4.0 \times 10^{-9} n_{0}$ & $2.5 \times 10^{-5} n_{0}$ & $6.6 \times 10^{-4} n_{0}$ & $6.4 \times 10^{-3} n_{0}$ \\
\hline$F_{\mathrm{H}}^{\beta^{0}}\left(V_{S}, n_{0}\right)\left(\operatorname{ergs~s}^{-1} \mathrm{~cm}^{-2}\right) \ldots \ldots$ & & $8.7 \times 10^{-5} n_{0}$ & $2.3 \times 10^{-4} n_{0}$ & $4.4 \times 10^{-4} n_{0}$ & $7.6 \times 10^{-4} n_{0}$ \\
\hline $\mathscr{R}_{[\mathrm{O \amalg I}] / \mathrm{H} \beta}\left(V_{S}\right) \ldots \ldots \ldots \ldots \ldots \ldots \ldots$ & & $1.52-1.58$ & $5.9-6.5$ & $8.0-9.1$ & $12.5-13.8$ \\
\hline 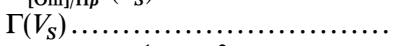 & & $9.2 \times 10^{-5}$ & $5.6 \times 10^{-2}$ & 0.55 & 2.0 \\
\hline \multirow{2}{*}{$F_{X}\left(\operatorname{ergs~s}^{-1} \mathrm{~cm}^{-2}\right) \ldots \ldots \ldots \ldots \ldots$} & “Nuclear jet" & $6.5 \times 10^{-18}$ & $4.0 \times 10^{-15}$ & $3.9 \times 10^{-14}$ & $1.4 \times 10^{-13}$ \\
\hline & S-shaped & $4.7 \times 10^{-18}$ & $2.9 \times 10^{-15}$ & $2.8 \times 10^{-14}$ & $1.0 \times 10^{-13}$ \\
\hline
\end{tabular}

NoTE.-The four upper rows list, for shock velocities of $200,300,400$ and $500 \mathrm{~km} \mathrm{~s}^{-1}$, the theoretical $0.1-2.4 \mathrm{keV}$ flux per unit of shock surface $\left(\Phi_{X}\right)$, including the effects of photoelectric absorption by an equivalent hydrogen column density of $\mathrm{N}_{\mathrm{H}}=0.186 \times 10^{22} \mathrm{~cm}^{-2}$ hydrogen column density (appropriate to NGC 2110 ); the theoretical $\mathrm{H} \beta$ flux per unit of shock surface $\left(\Phi_{H \beta}^{0}\right)$; the theoretical range of [O III] $] 25007 / \mathrm{H} \beta$ ratios $\left(\mathscr{R}_{[\mathrm{O} \mathrm{m}] / \mathrm{H} \beta}\right)$ for magnetic parameters $B_{0} / n_{0}^{1 / 2}$ between 0 and $4 \mu \mathrm{G} \mathrm{cm}^{3 / 2}$; and the coefficient $\Gamma$ (see $\S 5.2 .3$ for details). We then list the predicted 0.1-2.4 keV fluxes for the "nuclear jet" and "S-shaped" emission-line features of NGC 2110 as a function of the shock velocity. 
$\mathrm{keV} \mathrm{X}$-ray flux over the absorbed compact X-ray source of NGC 2110 originates from the extended component (Weaver et al. 1995), suggesting that very little hot, shocked gas is present closer to the nucleus, in the "nuclear jet." One remaining possibility is that the soft X-rays from hot gas close to the nucleus are photoelectrically absorbed, so such $\mathrm{X}$-rays are only seen when the hot gas has expanded to the observed radius of $4^{\prime \prime}[600 \mathrm{pc}]$.

\section{NGC 5929: DISCUSSION}

In NGC 5929, our 0".43 (69 pc) FOS aperture contains part of the southwest emission-line cloud (cloud A, B94; see Fig. 1). The line emission in cloud A is redshifted relative to the systemic velocity of the galaxy (Whittle et al. 1986; Wakamatsu \& Nishida 1988; Wilson \& Keel 1989; Ferruit et al. 1997b) and is morphologically associated with the southwest radio component (Ulvestad \& Wilson 1984; B94; $\mathrm{Su}$ et al. 1996; see Lattanzi et al. 1997 for an accurate optical-to-radio registration for NGC 5929). As before, we will compare the UV and optical line ratios derived from our FOS spectra of cloud A to the line ratios predicted by the MB-IB models (B96; B97) and the photoionizing shock models (DS95; DS96).

\subsection{MB-IB Central Source Photoionization Models}

Although the clear morphological association of cloud A with one of the radio components (B94; Lattanzi et al. 1997) and the observed line widths $\left(185 \pm 20 \mathrm{~km} \mathrm{~s}^{-1}\right.$ [O III] $\lambda 5007$ FWHM, Whittle et al. 1986; $220 \mathrm{~km} \mathrm{~s}^{-1}$ [N II] and $\mathrm{H} \alpha$ FWHM, Ferruit et al. 1997b) suggest an interaction between the radio material and the ISM in cloud A, there is, to date, no compelling evidence against central source photoionization of cloud A. Recent observations of $\mathrm{H} \mathrm{I}$ absorption toward the northeast radio lobe of NGC 5929 suggest that both the neutral and ionized gas undergo galactic rotation (Cole et al. 1998), behavior consistent with the gaseous kinematics expected in the frame of the central source photoionization scenario. Thus, we will compare the

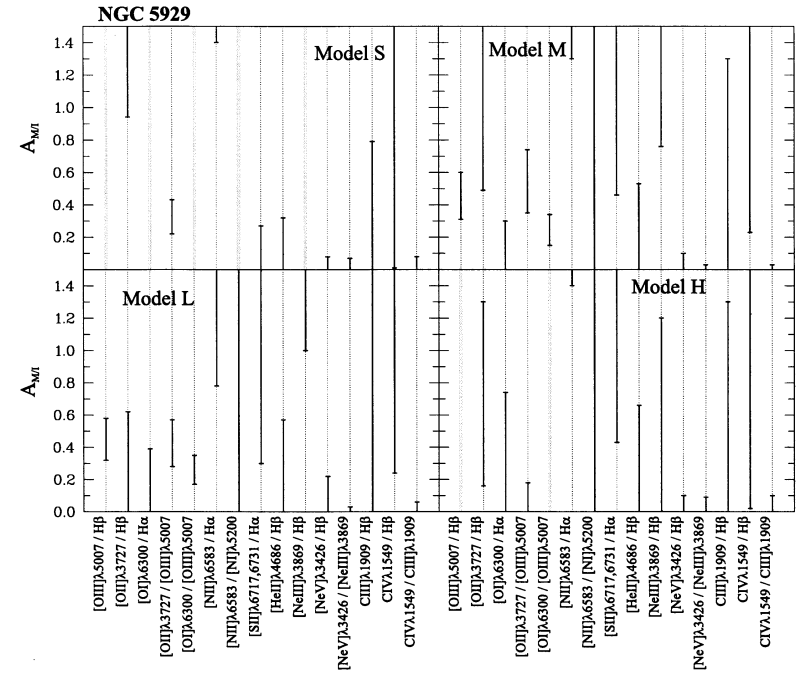

FIG. 8.- Ranges of $A_{M / I}$ obtained using the procedure described in $\S 5.1$, for a set of line ratios observed in NGC 5929, and for the four considered MB-IB models (S, L, M, and H). A gray line is displayed whenever an observed line ratio cannot be reproduced by a model.

observed line ratios with those predicted by the MB-IB models of B96, which are "pure" photoionization models.

Following the same procedure as before (see $\S 5.1$ ), we have computed the ranges of $A_{M / I}$ (from the solar abundance MB-IB models S [B96], L, M, and H [B97]) that correspond to the range of reddening-corrected $\left(A_{V}=0-1.2\right.$ mag) line ratios derived from our FOS spectra of cloud A. The results of this procedure are listed in Table 9 and displayed in Figure 8.

We can first rule out models $\mathrm{S}$ and $\mathrm{H}$ since they cannot reproduce the observed $[\mathrm{O} \mathrm{III}] / \mathrm{H} \beta$ ratio and give inconsistent ranges of $A_{M / I}$ for the [O II] $\lambda 3727 / \mathrm{H} \beta$ and [O II] $\lambda 3727 /[\mathrm{O}$ III $] \lambda 5007$ line ratios (there is in fact a very narrow overlap for model $\mathrm{H}$ ). For models $\mathrm{L}$ and $\mathrm{M}$, these three line ratios suggest $A_{M / I}$ values in the ranges $0.3-0.6$ (model L)

TABLE 9

Various Line ratios ObServed in NGC 5929 with Their Corresponding Values of $A_{M / I}$ For Four MB-IB MODELS (MODEL S, B96; MODELS L, M AND H, B97)

\begin{tabular}{|c|c|c|c|c|c|}
\hline \multirow[b]{2}{*}{ LINES } & \multirow[b]{2}{*}{ OBSERVED LINE RATIOS } & \multicolumn{4}{|c|}{$A_{M / I}$ RANGES } \\
\hline & & Model S & Model L & Model M & Model H \\
\hline$[\mathrm{O}$ iII $] \lambda 5007 / \mathrm{H} \beta \ldots$. & $2.1-3.5$ & $*$ & $0.32-0.58$ & $0.31-0.60$ & $*$ \\
\hline$[\mathrm{O}$ II $] \lambda 3727 / \mathrm{H} \beta \ldots \ldots \ldots \ldots \ldots \ldots . . .$. & $3.4-5.1$ & $2.3-0.94$ & $<0.62$ & $1.5-0.49$ & $1.3-0.16$ \\
\hline$\left[\mathrm{O}_{\mathrm{I}}\right] \quad \lambda 6300 / \mathrm{H} \alpha \ldots \ldots \ldots \ldots \ldots . . . . .$. & $0.30-0.52$ & $*$ & $<0.39$ & $<0.30$ & $<0.74$ \\
\hline$[\mathrm{O}$ II] $\lambda 3727 /[\mathrm{O}$ III $] \lambda 5007 \ldots \ldots .$. & $1.0-2.0$ & $0.43-0.22$ & $0.57-0.28$ & $0.74-0.35$ & $<0.18$ \\
\hline$[\mathrm{O}$ I $] \lambda 6300 /[\mathrm{O} \mathrm{III}] \lambda 5007 \ldots \ldots \ldots$ & $0.39-0.77$ & $*$ & $0.35-0.17$ & $0.34-0.15$ & $*$ \\
\hline$[\mathrm{N}$ II $] \lambda 6583 / \mathrm{H} \alpha \ldots \ldots \ldots \ldots \ldots \ldots . . . . .$. & $0.42-0.75$ & $4.0-1.4$ & $3.8-0.78$ & $4.6-1.3$ & $4.2-1.4$ \\
\hline$[\mathrm{N}$ II $] \lambda 6583 /[\mathrm{N} \mathrm{I}] \bar{\lambda} 5200 \ldots \ldots \ldots .$. & $3-16$ & $*$ & $<101$ & $<143$ & $<1300$ \\
\hline$[\mathrm{S}$ II $] \lambda \lambda 6717,6731 / \mathrm{H} \alpha \ldots \ldots \ldots \ldots$ & $0.53-0.85$ & $<0.27$ & $2.3-0.30$ & $1.8-0.46$ & $2.1-0.43$ \\
\hline He II $\lambda 4686 / \mathrm{H} \beta \ldots \ldots \ldots \ldots \ldots \ldots . . . . .$. & $<0.14$ & $<0.32$ & $<0.57$ & $<0.53$ & $<0.66$ \\
\hline$[\mathrm{Ne}$ III $] \lambda 3869 / \mathrm{H} \beta \ldots \ldots \ldots \ldots \ldots \ldots$ & $0.37-0.92$ & $*$ & $1.0-8.7$ & $0.76-33$ & $<1.2$ \\
\hline$[\mathrm{Ne} \mathrm{v}] \lambda 3426 / \mathrm{H} \beta \ldots \ldots \ldots \ldots \ldots$ & $<0.13$ & $<0.08$ & $<0.22$ & $<0.10$ & $<0.10$ \\
\hline$[\mathrm{Ne} \mathrm{v}] \lambda 3426 /[\mathrm{Ne}$ III $] \lambda 3869 \ldots \ldots$ & $<0.17$ & $<0.07$ & $<0.03$ & $<0.03$ & $<0.09$ \\
\hline $\mathrm{C}$ iv $\lambda 1549 / \mathrm{H} \beta \ldots \ldots \ldots \ldots \ldots \ldots \ldots$ & $<3.9$ & $<0.79$ & $<4.1$ & $<1.3$ & $<1.3$ \\
\hline $\mathrm{C}$ III] $\lambda 1909 / \mathrm{H} \beta \ldots \ldots \ldots \ldots \ldots \ldots$ & $0.4-3.7$ & $0.01-10.5$ & $0.24-12.9$ & $0.23-33$ & $>0.02$ \\
\hline $\mathrm{C}$ iv $\lambda 1549 / \mathrm{C}$ III] $\lambda 1909 \ldots \ldots \ldots .$. & $<1$ & $<0.08$ & $<0.06$ & $<0.03$ & $<0.1$ \\
\hline
\end{tabular}

Note. $-A_{M / I}$ : ratio of the solid angle subtended by the MB component to the solid angle subtended by the IB component. The listed ranges of observed ratios correspond to the allowed range of obscuration $\left(0<A_{V}<1.2 \mathrm{mag}\right)$. The symbol * is given when the observed ratio cannot be reproduced by the model. The ratio of the $\mathrm{H} \beta$ luminosity of the MB component to that of the IB component, $C_{M / I}$, is $0.568,0.354,0.368$ and 0.461 for the models $\mathrm{S}, \mathrm{L}, \mathrm{M}$ and $\mathrm{H}$, respectively (B96, B97). 
and $0.5-0.6$ (model $\mathrm{M})$. The $\left[\mathrm{O}_{\mathrm{I}}\right] \lambda 6300$ line ratios indicate lower values of $A_{M / I}$ in the ranges $0.17-0.35$ and $0.15-0.30$ for models $\mathrm{L}$ and $\mathrm{M}$, respectively. However, these values depend strongly on the extent partially ionized zone in the IB component (see $\S 5.1$ ).

The first strong discrepancy for models $\mathrm{L}$ and $\mathrm{M}$ comes from the $[\mathrm{N} \mathrm{II}] \lambda 6583 / \mathrm{H} \alpha$ ratio, which has relatively low observed values $(0.42-0.75)$, indicating a higher value of $A_{M / I}$ than suggested by the oxygen lines. The [N II] $\lambda 6583$ emission of the IB component could be smaller if the IB clouds were truncated earlier, but this would worsen the problem with the $\left[\mathrm{O}_{\mathrm{I}}\right] \lambda 6300$ line, which has the opposite requirement, as noted above. This could also be an abundance problem and would require nitrogen abundances lower than solar. The ranges of $A_{M / I}$ indicated by the [S II] $\lambda \lambda 6717,6731 / \mathrm{H} \alpha$ and the $\mathrm{He} \mathrm{II} / \mathrm{H} \beta$ ratios for models $\mathrm{L}$ and $\mathrm{M}$ are in good agreement with those obtained from the oxygen lines. However, both models $\mathrm{L}$ and $\mathrm{M}$ (in fact, all four MB-IB models) fail to reproduce the strengths of the neon lines in a consistent way.

As in the case of NGC 2110, the utility of the UV carbon lines is limited by the uncertainty in the reddening determination. The upper limits on $A_{M / I}$ derived from the C IV $\lambda 1549 / \mathrm{H} \beta$ ratio are high and do not provide any real constraint. The observed C III] $\lambda 1909 / \mathrm{H} \beta$ ratio permits a very broad range of $A_{M / I}$ in models $\mathrm{L}$ and $\mathrm{M}$, a range that is consistent with the values derived from the oxygen lines. This is not the case for the C IV $\lambda 1549 / C$ III] $\lambda 1909$ ratio which gives low $A_{M / I}$ values. Given the fact that both the C III] $\lambda 1909$ and C IV $\lambda 1549$ lines are emitted in the MB component (see Table 1 in B97), these low $A_{M / I}$ values are not affected by the IB component and indicate that the theoretical C IV $\lambda 1549 / \mathrm{C}$ III] $\lambda 1909$ ratio of the MB component is too high compared to the observed ratio. As discussed in $\S 5.2 .1$, this could be due to an overcorrection of the reddening of the $\mathrm{C} \mathrm{III]} \lambda 1909$ lines, as they are affected by the $2175 \AA$ extinction bump included in the extinction curve of Cardelli et al. (1989).

To summarize, the MB-IB models L and $\mathrm{M}$ (B97) approximately reproduce the observed line ratios for $A_{M / I}$ ranges of $0.3-0.6$ and $0.5-0.6$, respectively. The only strong discrepancies between the predictions of the models and the data are for the $[\mathrm{N} \mathrm{II}] / \mathrm{H} \alpha$ ratio and for the neon lines. Models S (B96) and H (B97) can be ruled out as they fail to reproduce the oxygen line ratios.

\subsection{Photoionizing Shock Models}

\subsubsection{Line Ratios}

We now compare the observed line ratios from our FOS data with those predicted by the fast, photoionizing shock models of DS95 and DS96. The observed [O II] $23727 /$ [O III] $\lambda 5007$ ratio of $1-2$ (for $A_{V}=0-1.2 \mathrm{mag}$ ) cannot be obtained with shock-only (no precursor) models (DS95, their Fig. 1), so we consider only models with both shock and precursor. The reddening-corrected ratios for NGC 5929 (for $A_{V}=0-1.2 \mathrm{mag}$ ) and the theoretical ratios for shock + precursor associations with shock velocities of $V_{S}=200,300,400$, and $500 \mathrm{~km} \mathrm{~s}^{-1}$, are listed in Table 10 .

Both the observed [O III] $\lambda 5007 / \mathrm{H} \beta(2.1-3.5)$ and [O II] $\lambda 3727 /[\mathrm{O}$ III $] \lambda 5007(1-2)$ ratios in cloud $\mathrm{A}$ indicate a low shock velocity, between 200 and $300 \mathrm{~km} \mathrm{~s}^{-1}$. Little information on $V_{S}$ can be extracted from the observed [O II] $\lambda 3727 /$ $\mathrm{H} \beta$ ratio (3.4-5.1), which can be reproduced for all velocities higher than $200 \mathrm{~km} \mathrm{~s}^{-1}$ by varying the magnetic parameter $B_{0} / n_{0}^{1 / 2}$ in the model. The observed [O I] $\lambda 6300 / \mathrm{H} \alpha(0.30$ 0.52 ) is consistent with a $300 \mathrm{~km} \mathrm{~s}^{-1}$ shock but is slightly too high for shock velocities below $300 \mathrm{~km} \mathrm{~s}^{-1}$. In the same way, the [ [ $\mathrm{O}$ I] $\lambda 6300 /\left[\begin{array}{ll}\mathrm{O} & \mathrm{III}\end{array} \lambda 5007\right.$ ratio $(0.39-0.77)$ observed in cloud A is too high and cannot be reproduced by the models, which predict ratios within a roughly constant range (0.15-0.30). This suggests that the photoionizing shock models underpredict the strength of the $\left[\mathrm{O}_{\mathrm{I}}\right] \lambda 6300$ line. No constraint can be derived from the lower limit on the temperature sensitive $[\mathrm{O}$ III $] \lambda 5007 /[\mathrm{O}$ III $] \lambda 4363$ ratio.

The ratio of the $[\mathrm{N} \mathrm{II}] \lambda 6583$ line to $\mathrm{H} \alpha$ is also consistent with a $200-300 \mathrm{~km} \mathrm{~s}^{-1}$ shock velocity although, as for [O I] $\lambda 6300 / \mathrm{H} \alpha$, the observed range tends to be higher than the predicted one. This problem is more acute for the [S II] $\lambda \lambda 6717,6731 / \mathrm{H} \alpha$ ratio, which is barely reached for a 300 $\mathrm{km} \mathrm{s}^{-1}$ shock and suggests higher shock velocities. Contrary to the MB-IB models, the fast-shock models have no

TABLE 10

LINE RATIOS IN NGC 5929

\begin{tabular}{|c|c|c|c|c|c|}
\hline Line Ratio & Observed & $V_{S}=200 \mathrm{~km} \mathrm{~s}^{-1}$ & $V_{S}=300 \mathrm{~km} \mathrm{~s}^{-1}$ & $V_{S}=400 \mathrm{~km} \mathrm{~s}^{-1}$ & $V_{S}=500 \mathrm{~km} \mathrm{~s}^{-1}$ \\
\hline$[\mathrm{O} \mathrm{III}] \quad \lambda 5007 / \mathrm{H} \beta \ldots \ldots \ldots \ldots \ldots \ldots$ & $2.1-3.5$ & $1.52-1.58$ & $5.9-6.5$ & $8.0-9.1$ & $12.5-13.8$ \\
\hline$[\mathrm{O}$ II $] \lambda 3727 / \mathrm{H} \beta \ldots \ldots \ldots \ldots \ldots \ldots . . . .$. & $3.4-5.1$ & $2.3-3.6$ & $1.7-5.2$ & $1.7-7.0$ & $1.7-7.2$ \\
\hline$\left[\mathrm{O}_{\mathrm{I}}\right] \quad \lambda 6300 / \mathrm{H} \alpha \ldots \ldots \ldots \ldots \ldots . . . .$. & $0.30-0.52$ & $0.07-0.13$ & $0.32-0.47$ & $0.57-0.81$ & $0.81-0.95$ \\
\hline$[\mathrm{O}$ II $] \lambda 3727 /[\mathrm{O}$ III $] \lambda 5007 \ldots \ldots .$. & $1-2$ & $1.5-2.3$ & $0.3-0.8$ & $0.2-0.8$ & $0.1-0.5$ \\
\hline$[\mathrm{O}$ I $] \lambda 6300 /[\mathrm{O}$ III $] \lambda 5007 \ldots \ldots \ldots$ & $0.39-0.77$ & $0.14-0.26$ & $0.15-0.23$ & $0.18-0.30$ & $0.17-0.21$ \\
\hline$[\mathrm{O} \mathrm{III}] \lambda 5007 /[\mathrm{O} \mathrm{III}] \lambda 4363 \ldots \ldots .$. & $>23$ & $\sim 28$ & $103-108$ & $107-109$ & $79-82$ \\
\hline$[\mathrm{N}$ II $] \lambda 6583 / \mathrm{H} \alpha \ldots \ldots \ldots \ldots \ldots \ldots . . . .$. & $0.42-0.75$ & $0.19-0.41$ & $0.23-0.65$ & $0.34-0.86$ & $0.39-0.85$ \\
\hline$[\mathrm{S}$ II $] \lambda \lambda 6717,6731 / \mathrm{H} \alpha \ldots \ldots \ldots \ldots$ & $0.53-0.85$ & $0.18-0.32$ & $0.41-0.56$ & $0.48-0.75$ & $0.49-0.78$ \\
\hline He II $\lambda 4686 / \mathrm{H} \beta \ldots \ldots \ldots \ldots \ldots \ldots$ & $<0.14$ & $\sim 0.05$ & $0.23-0.28$ & $0.28-0.37$ & $0.23-0.31$ \\
\hline$[\mathrm{Ne}$ III $] \lambda 3869 / \mathrm{H} \beta \ldots \ldots \ldots \ldots \ldots . .$. & $0.37-0.92$ & $0.18-0.21$ & $0.50-0.72$ & $0.71-1.1$ & $1.2-1.6$ \\
\hline$[\mathrm{Ne} v] \lambda 3426 / \mathrm{H} \beta \quad \ldots \ldots \ldots \ldots \ldots$ & $<0.13$ & $\sim 0.13$ & $\sim 0.10$ & $\sim 0.12$ & $\sim 0.4$ \\
\hline$[\mathrm{Ne} \mathrm{v}] \lambda 3426 /[\mathrm{Ne}$ III] $\lambda 3869 \ldots \ldots$ & $<0.17$ & $0.64-0.73$ & $0.14-0.21$ & $0.11-0.18$ & $0.25-0.37$ \\
\hline $\mathrm{C}$ IV $\lambda 1549 / \mathrm{H} \beta \ldots \ldots \ldots \ldots \ldots \ldots$ & $<3.9$ & $\sim 2.1$ & $1.0-1.1$ & $1.1-1.2$ & $3.3-3.7$ \\
\hline $\mathrm{C}$ III] $\lambda 1909 / \mathrm{H} \beta \ldots \ldots \ldots \ldots \ldots \ldots$ & $0.4-3.7$ & $0.33-0.38$ & $0.22-0.34$ & $0.49-0.77$ & $1.1-1.4$ \\
\hline $\mathrm{C}$ iv $\lambda 1549 / \mathrm{C}$ III] $\lambda 1909 \ldots \ldots \ldots . .$. & $<2$ & $5.6-6.3$ & $3.1-4.9$ & $1.5-2.4$ & $2.5-3.3$ \\
\hline
\end{tabular}

NoTE.-The second column gives the observed range (allowing for the uncertainty in obscuration- $A_{V}=0-1.2$ mag), while the third, fourth, fifth, and sixth columns give the ranges predicted by the photoionizing shock models of DS95 and DS96 (shock + precursor) for four shock velocities, $V_{S}=200,300,400$, and $500 \mathrm{~km} \mathrm{~s}^{-1}$. The range given corresponds to a range of "magnetic parameters," $B / n_{0}^{1 / 2}$, from 0 to 4 $\mu \mathrm{G} \mathrm{cm}{ }^{3 / 2}$. 
problem reproducing the neon line ratios. Once more, the expected line ratios for shock velocities around $300 \mathrm{~km} \mathrm{~s}^{-1}$ are in good agreement with the observations.

As for NGC 2110, the uncertainties in the reddening result in very large error bars on the observed ultraviolet carbon line ratios. The high upper limit on the ultraviolet $\mathrm{C}$ IV $\lambda 1549$ line flux relative to $\mathrm{H} \beta$ derived from our observations is too high to be used to put any constraints on the model parameters. The $\mathrm{C} \mathrm{III]} \lambda 1909 / \mathrm{H} \beta$ ratios predicted for a $300 \mathrm{~km} \mathrm{~s}^{-1}$ shock (0.22-0.34) are lower than the observed range of ratios (0.4-3.7), while the predicted C IV $\lambda 1549$ / C III] $\lambda 1909$ ratio is higher (3.1-4.9) than the observed upper limit $(<2)$. These observed UV line ratios are therefore more representative of faster shocks, with velocities of $400-500 \mathrm{~km} \mathrm{~s}^{-1}$. This could indicate that there is a whole range of shock velocities in our aperture, or, as noted in $\S 5.2 .1$, this might be a consequence of an overcorrection of the reddening at the wavelengths of $\mathrm{C} \mathrm{III]} \lambda 1909$.

Therefore, on average, the observed spectrum of cloud A is correctly reproduced by photoionizing shock models with velocities $\simeq 300 \mathrm{~km} \mathrm{~s}^{-1}$, although they tend to predict strengths that are too low for the low-excitation lines. There could also be a problem with the C III] $\lambda 1909$ lines, but this might be related to uncertainty in the reddening curve near their wavelength.

\subsubsection{Preshock Density and Shock Surface}

Using the shock velocity of $300 \mathrm{~km} \mathrm{~s}^{-1}$ determined above and the same method as in $\S 5.2 .2$, we can estimate other parameters of the shock models - the preshock density, the shock surface area, and the volume of the shock + precursor structure. First, the compression factors appropriate to the [S II]-emitting zone are computed from Tables 8-11 in DS96 and are 26, 92, 130, and 725 for magnetic parameters $B_{0} / n_{0}^{1 / 2}$ of $4,2,1$, and $0 \mu \mathrm{G} \mathrm{cm} / 2$, respectively. Given the density derived from the $[\mathrm{S}$ II] ratio $\left(320_{-150}^{+220} \mathrm{~cm}^{-3}\right)$, this gives preshock densities in the (broad) range $n_{0}=0.23-20.8 \mathrm{~cm}^{-3}$. Then, we can express the shock surface area, $\mathscr{S}_{\text {shock}}$ as a function of the absolute (reddening-corrected) $\mathrm{H} \beta$ luminosity in our aperture, $\mathscr{L}_{\mathrm{H} \beta}$, and the preshock density, $n_{0}$, in an equation analogous to equation (3) but appropriate to a $300 \mathrm{~km} \mathrm{~s}^{-1}$ shock :

$$
\mathscr{S}_{\text {shock }}\left[\mathrm{cm}^{2}\right]=\frac{1}{2.26 \times 10^{-4}} \frac{\mathscr{L}_{\mathrm{H} \beta}\left[\mathrm{ergs} \mathrm{s}^{-1}\right]}{n_{0}\left[\mathrm{~cm}^{-3}\right]} .
$$

For $A_{V}$ between 0.6 and $1.2 \mathrm{mag}$, the reddening-corrected $\mathrm{H} \beta$ flux in our aperture ranges from 7.3 to $18.1 \times 10^{-15}$ ergs s${ }^{-1} \mathrm{~cm}^{-2}$. Assuming a distance to NGC 5929 of 34 $\mathrm{Mpc}$, this gives absolute $\mathrm{H} \beta$ luminosities in our aperture between 1.0 and $2.5 \times 10^{39} \mathrm{ergs} \mathrm{s}^{-1}$. Using these values of $\mathscr{L}_{\mathrm{H} \beta}$ together with our estimates of $n_{0}\left(0.23-20.8 \mathrm{~cm}^{-3}\right)$ in equation (6), we obtain shock surface areas between 0.02 and $5 \mathrm{kpc}^{2}$, i.e., between $150 \mathrm{pc} \times 150 \mathrm{pc}\left(0^{\prime \prime} .9 \times 0.9\right)$ and $2.2 \mathrm{kpc} \times 2.2 \mathrm{kpc}\left(14^{\prime \prime} \times 14^{\prime \prime}\right)$.

We also derive a lower limit on the volume of the shock + precursor structure $\left(\mathscr{V}_{s+p}\right)$. For a $300 \mathrm{~km} \mathrm{~s}^{-1}$ shock, the thickness of the $\mathrm{H}$ II region of the precursor is $L_{p}=261\left(n_{0}\left[\mathrm{~cm}^{-3}\right]\right)^{-1}$ pc. This gives (analogous to eq. [4], but appropriate to a $300 \mathrm{~km} \mathrm{~s}^{-1}$ shock)

$$
\mathscr{V}_{s+p}\left[\mathrm{pc}^{3}\right]>1.21 \times 10^{-31} \frac{\mathscr{L}_{\mathrm{H} \beta}\left[\mathrm{ergs} \mathrm{s}^{-1}\right]}{\left(n_{0}\left[\mathrm{~cm}^{-3}\right]\right)^{2}} .
$$

For our minimum and maximum estimated values of the preshock density, this yields $\mathscr{V}_{s+p}>2-6 \times 10^{9} \mathrm{pc}^{3}\left(n_{0}=\right.$ $\left.0.23 \mathrm{~cm}^{-3}\right)$ and $\mathscr{V}_{s+p}>0.3-0.7 \times 10^{6} \mathrm{pc}^{3}\left(n_{0}=34 \mathrm{~cm}^{-3}\right)$. Assuming that the ionized gas region in our aperture has a thickness of $\simeq 300 \mathrm{pc}$ (typical scale height of the $\mathrm{H} \mathrm{I}$ in a galaxy disk; Dickey \& Lockman 1990), the volume included in our aperture is only $\simeq 1.1 \times 10^{6} \mathrm{pc}^{3}$. Therefore, as for NGC 2110, the actual preshock density must be near the high end of our estimates, the compression factor small and the magnetic parameter near the high end of the range considered.

\section{CONCLUSION}

We have reported $H S T / F O S$ spectra of extranuclear regions of line emission from 1090 to $6820 \AA$ in the two Seyfert 2 galaxies NGC 2110 and NGC 5929. In NGC 2110, the aperture ( $65 \mathrm{pc}$ diameter) included the brightest region of the "nuclear jet" emission-line feature (M94), while in NGC 5929, the aperture (69 pc diameter) contained the southwest emission-line cloud (cloud A; B94). In the UV, we have detected the Ly $\alpha$ (NGC 2110 only) and C III] $\lambda 1909$ lines, but not the C IV $\lambda 1549$ lines. Fluxes of the detected lines of astrophysical interest and upper limits on some other key lines have been measured and corrected for reddening. They have been compared with the predictions of the composite matter-bounded (MB) plus ionization bounded (IB) model clouds, which are photoionized by a central source (B96 and B97) and of fast-shock models which photoionize the gas "in situ" (DS95, DS96). In both objects, the relatively high and uncertain reddening inferred from the Balmer line ratios strongly weakens the utility of the UV carbon lines as diagnostics of the ionization process.

In the case of NGC 2110, the spectra are of relatively high excitation, the $[\mathrm{Ne} v] \lambda \lambda 3346,3426$ lines being detected. There is weak evidence that [O III] $\lambda 5007$ and $[\mathrm{N} \mathrm{II}] \lambda 6583$ are blueshifted with respect to the Balmer lines. Comparison of the reddening corrected, observed line ratios with four MB-IB models (model S, B96; models L, M, and H, B97) shows that models $\mathrm{L}, \mathrm{M}$, and $\mathrm{H}$ are ruled out, while model S agrees only approximately with the data. However, fine-tuning of some of the MB-IB model parameters, such as the ionizing continuum hardness and the truncation level of the ionization-bounded component, could significantly improve the match of these models to the data, and so they cannot be ruled out. A better match to the data is obtained for shock + precursor models with shock velocities $\simeq 400$ $\mathrm{km} \mathrm{s}^{-1}$. For this velocity, we infer preshock densities in the range $0.9-34 \mathrm{~cm}^{-3}$. Imposing the requirement that the total volume of gas in the shock models must be less than the volume included in our observing aperture implies that the preshock density must be near the upper end of this range, the compression factor small, and the magnetic parameter near the high end of the considered range. Comparison between the [O $\mathrm{III}] 25007$ luminosity of the northern emission-line features of NGC 2110 (the "nuclear jet" and "S-shaped" structures) and the extended soft X-ray emission detected north of the nucleus by Weaver et al. (1995) shows that, if the emission-line features are photoionizing shocks, shock velocities higher than $500 \mathrm{~km} \mathrm{~s}^{-1}$ are required to power the extended X-ray emission.

Cloud A in NGC 5929 exhibits spectral characteristics of lower excitation than what is seen in NGC 2110, with particularly strong hydrogen Balmer lines. No shift or profile change between different emission lines is detected. In this 
object, the MB-IB models provide once more a relatively poor match to the data, particularly to the strengths of the neon lines, whereas photoionizing shock models with velocities $\simeq 300 \mathrm{~km} \mathrm{~s}^{-1}$ correctly reproduce most of the observed line ratios. Assuming a shock velocity of $300 \mathrm{~km}$ $\mathrm{s}^{-1}$, we infer preshock densities for the photoionizing shock models of $0.23-20.8 \mathrm{~cm}^{-3}$ and find that a total shock surface area in the range $0.02-5 \mathrm{kpc}^{2}$ is required to power the line emission in our aperture. As for NGC 2110, the observationally inferred line power and emitting volume imply that the preshock density must be near the high end of the above range, the compression factor small, and the magnetic parameter near the high end of the range considered.

In the future, high spatial resolution observations of the gaseous kinematics in these objects with $H S T$, and high spatial resolution X-ray observations with the $A X A F$ satellite, should be able to distinguish between the central source and "in situ" shock photoionization models. In particular, the photoionizing shock models make clear predictions concerning the soft X-ray emissions of these extranuclear gas clouds (cf. Wilson \& Raymond 1999).

We are very grateful to Anuradha Koratkar for her assistance ("above and beyond the call of duty") in planning and executing these observations. P. F. thanks Gary Bower for sending his deconvolved $H S T / \mathrm{WF} / \mathrm{PC}$ continuum and emission-line images of NGC 5929, as well as Ed Smith and Anuradha Koratkar for their help in reducing the FOS data. P. F. has made extensive use of the multipurpose photoionization code Mappings Ic (Ferruit et al. 1997a) kindly provided by Luc Binette. This research was supported by NASA, STScI, and the National Science Foundation through grants NAG81027, GO6006, and AST 9527289, respectively. It has made use of the NASA's Astrophysics Data System Abstract Service.
Aldrovandi, S. M. V., \& Contini, M. 1985, A\&A, 149, 109

Allen, M. G., Dopita, M. A., \& Tsvetanov, Z. I. 1998, ApJ, 493, 571

Arnaud, K. A. 1996, in ASP Conf. Ser. 101, Astronomical Data Analysis Software and Systems V, ed. G. Jacoby \& J. Barnes (San Francisco: ASP), 17

Arp, H. 1966, Atlas of Peculiar Galaxies (Pasadena: California Inst. of Tech.)

Axon, D. J., Marconi, A., Capetti, A., Maccheto, F. D., Schreier, E., \& Robinson, A. 1998, ApJ, 496, 75

Binette, L., Calvet, N., Cantó, J., \& Raga, A. C. 1990, PASP, 102, 723

Binette, L., Dopita, M. A., \& Tuohy, I. R. 1985, ApJ, 297, 476

Binette, L., Wilson, A. S., Raga, A., \& Storchi-Bergmann, T. 1997, A\&A, 327, 909 (B97)

Binette, L., Wilson, A. S., \& Storchi-Bergmann, T. 1996, A\&A, 312, 365 (B96)

Bower, G. A., Wilson, A. S., Mulchaey, J. S., Miley, G. K., Heckman, T. M., \& Krolik, J. H. 1994, AJ, 107, 1686 (B94)

Bradt, H. V., Burke, B. F., Canizares, C. R., Greenfield, P. E., Kelley, R. L., McClintock, J. E., van Paradijs, J., \& Koski, A. T. 1978, ApJ, 226, L111 Burstein, D., \& Heiles, C. 1982, AJ, 87, 1165

. 1984, ApJS, 54, 33

Capetti, A., Axon, D. J., Macchetto, F., Spark, W. B., \& Boksenberg, A. 1996, ApJ, 469, 554

Cardelli, J. A., Clayton, G. C., \& Mathis, J. S. 1989, ApJ, 345, 245

Cole, G. H. J., Pedlar, A., Mundell, C. G., Gallimore, J. F., \& Holloway, A. J. 1998, MNRAS, 301, 782

Contini, M. 1997, A\&A, 323, 71

Contini, M., \& Aldrovandi, S. M. V. 1983, A\&A, 127, 15

Dickey, J. M., \& Lockman, F. J. 1990, ARA\&A, 28, 215

Dopita, M. A., Koratkar, A. P., Allen, M. G., Tsvetanov, Z. I., Ford, H. C.,

Bicknell, G. V., \& Sutherland, R. S. 1997, ApJ, 490, 202

Dopita, M. A., \& Sutherland, R. S. 1995, ApJ, 455, 468 (DS95)

. 1996, ApJS, 102, 161 (DS96)

Falcke, H., Wilson, A. S., \& Simpson, C. 1998, ApJ, 502, 199

Falcke, H., Wilson, A. S., Simpson, C., \& Bower, G. A. 1996, ApJ, 470, L31

Ferland, G. J., \& Osterbrock, D. E. 1986, ApJ, 300, 658

Ferruit, P., Binette, L., Sutherland, R. S., \& Pécontal, E. 1997a, A\&A, 322, 73

Ferruit, P., Pécontal, E., Wilson, A. S., \& Binette, L. 1997b, A\&A, 328, 493

Ferruit, P., Wilson, A. S., Falcke, H., Simpson, C., Pécontal, E., \& Durret, F. 1999, MNRAS, in press

Gaskell, C. M., \& Ferland, G. J. 1984, PASP, 96, 393

Halpern, J. P., \& Steiner, J. E. 1983, ApJ, 269, L37

\section{EFERENCES}

Haniff, C. A., Ward, M. J., \& Wilson, A. S. 1991, ApJ, 368, 167

Haniff, C. A., Wilson, A. S. \& Ward, M. J. 1988, ApJ, 334, 104

Hayashi, I., Koyama, K., Awaki, H., Ueno, S., \& Yamauchi, S. 1996, PASJ, 48,219

Keel, W. C., Kennicut, R. C., Jr., Hummel, E., \& van der Hulst, J. M. 1985, AJ, 90,708

Keyes, C. D., Koratkar, A. P., Dahlem, M., Hayes, J., Christensen, J., \& Martin, S. 1995, FOS Instrument Handbook v6.0

Lattanzi, M. G., Capetti, A., \& Macchetto, F. D. 1997, A\&A, 318, 997

Leitherer, C., et al. 1996, PASP, 108, 996

McClintock, J. E., van Paradijs, J., Remillard, R. A., Canizares, C. R., Koski, A. T., \& Véron, P. 1979, ApJ, 233, 809

Morse, J. A., Cecil, G., Wilson, A. S., \& Tsvetanov, Z. I. 1998, ApJ, 505, 159

Morse, J. A., Raymond, J. C., \& Wilson, A. S. 1996, PASP, 108, 426

Mulchaey, J. S., Wilson, A. S., Bower, G. A., Heckman, T. M., Krolik, J. H., \& Miley, G. K. 1994, ApJ, 433, 625 (M94)

Nagar, N., Wilson, A. S., Mulchaey, J. S., \& Gallimore, J. F. 1999, ApJS, 120,209

Nelson, C. H., \& Whittle, M. 1995, ApJS, 99, 67

Osterbrock, D. 1989, Astrophysics of Gaseous Nebulae and Active Galactic Nuclei (Mill Valley: University Science)

Pécontal, E., Ferruit, P., Binette, L., \& Wilson, A. S. 1997, Ap\&SS, 248, 167

Pogge, R. W. 1989, ApJ, 345, 730

Rousset, A. 1992, Ph.D. thesis, Univ. J. Monnet de Saint-Etienne

Shuder, J. M. 1980, ApJ, 240, 32

Storchi-Bergmann, T., Winge, C., Ward, M. J., \& Wilson, A. S. 1999, MNRAS, 304,35

Su, B. M., Muxlow, T. W. B., Pedlar, A., Holloway, A. J., Steffen, W., Kukula, M. J., \& Mutel, R. L. 1996, MNRAS, 279, 1111

Ulvestad, J. S., \& Wilson, A. S. 1984, ApJ, 285, 439

Viegas-Aldrovandi, S. M., \& Contini, M. 1989, ApJ, 339, 689

Wakamatsu, K.-I., \& Nishida, M. T. 1988, ApJ, 325, 596

Weaver, K. A., Mushotzky, R. F., Serlemitsos, P. J., Wilson, A. S., Elvis, M., \& Briel, U. 1995, ApJ, 442,597

Whittle, M., Haniff, C. A., Ward, M. J., Meurs, E. J. A., Pedlar, A., Unger, S. W., Axon, D. J., \& Harrison, B. A. 1986, MNRAS, 222, 189

Whittle, M., Pedlar, A., Meurs, E. J. A., Unger, S. W., Axon, D. J., \& Ward, M. J. 1988, ApJ, 326, 125

Wilson, A. S., Baldwin, J. A., \& Ulvestad, J. S. 1985, ApJ, 291, 627

Wilson, A. S., Binette, L., \& Storchi-Bergmann, T. 1997, ApJ, 482, L131

Wilson, A. S., \& Keel, W. C. 1989, 98, 1581

Wilson, A. S., \& Raymond, J. C. 1999, ApJ, 513, L115

Wilson, A. S., \& Willis, A. G. 1980, ApJ, 240, 429 